\title{
ANALYTIC CONTROLLABILITY OF THE WAVE EQUATION OVER A CYLINDER
}

\author{
Brice AlliberT ${ }^{1}$
}

\begin{abstract}
We analyze the controllability of the wave equation on a cylinder when the control acts on the boundary, that does not satisfy the classical geometric control condition. We obtain precise estimates on the analyticity of reachable functions. As the control time increases, the degree of analyticity that is required for a function to be reachable decreases as an inverse power of time. We conclude that any analytic function can be reached if that control time is large enough. In the $C^{\infty}$ class, a precise description of all reachable functions is given.
\end{abstract}

Résumé. On donne des estimations sur l'analycité nécessaire pour qu'une fonction soit contrôlable en temps fini sur un cylindre pour l'équation des ondes. Cette valeur décroit polynomialement avec $T$. On en déduit que toute fonction analytique peut être contrôlée en un temps assez grand. On donne de plus une description précise des fonctions $C^{\infty}$ qui sont contrôlables de cette façon.

AMS Subject Classification. 93B03, 93B05, 35L05, 35L20, 35B37.

Received September 8, 1997. Revised April 30, 1998.

\section{INTRODUCTION}

In this paper, we consider the control problem for the wave equation over a cylindrical surface. We denote this surface $C$ and we suppose, for the sake of simplicity, that its radius is 1 and its length $\pi$. We can parameterize $C$ in $\mathbb{R}^{3}$ by $x^{2}+y^{2}=1$ and $z \in(0, \pi)$. We will also denote $x=\cos \theta$ and $y=\sin \theta$ so that $(z, \theta) \in(0, \pi) \times \mathbb{S}^{1}$ is a set of coordinates over $C$.

The controlled part of the boundary will be $\Gamma=\partial C \cap\{z=0\}$. Thus the uniqueness time for this problem (i.e. the time that is needed to guarantee that a solution of the wave equation vanishing on $\Gamma$ with its normal derivative, vanishes everywhere) is $T_{u}=2 \pi$. As usual, we shall denote $E_{0}=H_{0}^{1}(C) \oplus L^{2}(C)$ and $E_{-1}=$ $L^{2}(C) \oplus H^{-1}(C)$.

The goal of this paper is to give results about the space $F_{T}$ of controlled functions in time $T>T_{u}$, e.g. the set of all functions $\underline{u}$ in $E_{-1}$ for which there exists a control $g(\theta, t)$ in $L^{2}(\Gamma \times(0, T))$ such that the solution of problem

$$
\mid \begin{aligned}
& \square u=0 \text { over } C \times(0, T) \\
& \left.\left(u, \partial_{t} u\right)\right|_{t=0}=\underline{u} \\
& \left.u\right|_{\partial C \times(0, T)}=g \mathbf{1}_{\Gamma \times(0, T)}
\end{aligned}
$$

Keywords and phrases: Wave equation, attainable set, analyticity, boundary control.

${ }^{1}$ CMAT, École Polytechnique, UMR 7640 du CNRS, F-91128 Palaiseau, France; e-mail: allibert@math.polytechnique.fr 
satisfies

$$
\left.\left(u, \partial_{t} u\right)\right|_{t=T}=0 .
$$

Let us introduce a few more notations in order to state our theorems. For any positive real number $\alpha$, we will denote

$$
\begin{aligned}
G_{0, \alpha} & =\left\{u(z, \theta)=\sum_{n, k} \alpha_{n, k} e^{-\alpha|n|} \sin k z e^{i n \theta} \mid\left(\alpha_{n, k}\right)_{n, k} \in l^{2}\right\} \\
G_{-1, \alpha} & =\left\{u(z, \theta)=\sum_{n, k} \alpha_{n, k} e^{-\alpha|n|} \sin k z e^{i n \theta} \mid\left(\frac{\alpha_{n, k}}{\sqrt{n^{2}+k^{2}}}\right)_{n, k} \in l^{2}\right\} \\
G_{\alpha} & =G_{0, \alpha} \times G_{-1, \alpha} .
\end{aligned}
$$

It is easily seen that if $\alpha^{\prime} \geq \alpha$ then $G_{\alpha^{\prime}} \subset G_{\alpha}$ and that $G_{0}=E_{-1}$.

Let us remark that all the elements of $G_{\alpha}$ are holomorphic on the complex band $|\Im m \theta|<\alpha$. Conversely, if a function $u(z, \theta)=\sum_{n, k} \alpha_{n, k} \sin k z e^{i n \theta}$ of $L^{2}\left((0, \pi) \times \mathbb{S}^{1}\right)$ is holomorphic on a band $|\Im m \theta|<\alpha+\epsilon$ for a positive $\epsilon$, then we can prove that it belongs to $G_{0, \alpha}$ and $G_{-1, \alpha}$.

Proof. Indeed, the function $v(x, \theta) \mapsto u(x, \theta+i \alpha)$ belongs to $L^{2}\left((0, \pi) \times \mathbb{S}^{1}\right)$. So the sequence $\left(\beta_{n, k}\right)$ of its Fourier coefficients belongs to $l^{2}(\mathbb{Z} \times \mathbb{N})$. Now by analyticity and periodicity,

$$
\begin{aligned}
\beta_{n, k} & =\int v(x, \theta) e^{-i n \theta} \sin k z d \theta d z \\
& =\int v(x, \theta-i \alpha) e^{-i n(\theta-i \alpha)} \sin k z d \theta d z \\
& =e^{-n \alpha} \int u(x, \theta) e^{-i n \theta} \sin k z d \theta d z \\
& =e^{-n \alpha} \alpha_{n, k} .
\end{aligned}
$$

So the sequence $\left(e^{-n \alpha} \alpha_{n, k}\right)$ belongs to $l^{2}$. For symmetric reasons, the sequence $\left(e^{+n \alpha} \alpha_{n, k}\right)$ also does. So $\left(e^{|n| \alpha} \alpha_{n, k}\right)$ belongs to $l^{2}$.

Hence

$$
u=\sum_{n, k} \gamma_{n, k} e^{-|n| \alpha} \sin k z e^{i n \theta},
$$

with $\left(\gamma_{n, k}\right) \in l^{2}(\mathbb{Z} \times \mathbb{N})$. So $u$ belongs $G_{0, \alpha}$. It is easy to see that it also belongs to $G_{-1, \alpha}$.

In order to give quantitative results about $F_{T}$, we introduce the value

$$
\alpha_{C}(T)=\inf \left\{\alpha \in \mathbb{R}^{+} \text {such that } G_{\alpha} \subset F_{T}\right\}
$$

We know (see [1] and [2]) that for any time $T>T_{u}$,

$$
\alpha_{C}(T) \leq \pi
$$


This means that any initial condition that can be continued as an holomorphic function with respect to $\theta$ over the band $|\Im m \theta| \leq \pi+\epsilon$ can be controlled in any time $T>T_{u}$.

In this paper, we will improve this result by proving the following two theorems

Theorem 1.1. For any positive number $\delta$, there is a constant $C_{\delta}$ such that for any time $T>T_{u}$,

$$
\alpha_{C}(T) \leq \frac{C_{\delta}}{T^{1-\delta}} .
$$

Theorem 1.2. There exists a constant $c$ such that for any time $T>T_{u}$,

$$
\alpha_{C}(T) \geq \frac{c}{T^{2}}
$$

Notice that Theorem 1.1 implies that any analytic initial condition belongs to some $F_{T}$ for $T$ large enough. Namely, if we denote $F_{\infty}=\bigcup_{T} F_{T}$,

$$
C^{\omega} \times C^{\omega} \subset F_{\infty}
$$

Melrose and Sjöstrand have proved that the analytic wave front of a solution of the wave equation propagates at the speed of light along the geodesics of the surface. (A simple definition of the analytic wave front set is given in Sect. 3.1.) In our case, it means that the analytic wave front of a solution of problem (1.1) will propagate until it reaches the boundary (at least).

The orthogonal sections of the cylindrical surface are geodesics that never hit the boundary. So if the analytic wave front set of the initial data of problem (1.1) contains a point on one of these geodesics, it will propagate, without ever hitting the boundary. So at any time $T,\left.u\right|_{t=T}$ or $\left.\partial_{t} u\right|_{t=T}$ will have at least one point in its analytic wave front. Therefore, it will never be 0 . Hence, no such function can belong to $F_{T}$, even if $T$ is very big. This means that these functions do not belong to $F_{\infty}$.

We will prove in this article that up to a periodisation of the problem that is needed to define the wave front at the boundary, these are the only functions of $C^{\infty}$ that do not belong to $F_{\infty}$, which gives us a geometric description of this space.

To be more precise, for any distribution $u(z, \theta)$ in $H^{-1}\left((0, \pi) \times \mathbb{S}^{1}\right)$, let us denote $P u$ the distribution in $\mathcal{D}^{\prime}\left(\mathbb{R} \times \mathbb{S}^{1}\right)$ obtained by putting first, for $z \in(0, \pi), u(\pi+z)=-u(\pi-z)$ and then by putting for any integer $k, u(z+2 k \pi)=u(z)$. Considering $P u$ allows us to see the singularities of $u$ on the boundary of the cylinder. Then we have the following theorem:

Theorem 1.3. If $\underline{u}$ belongs to $E_{-1}$, if $P \underline{u}$ belongs to $C^{\infty} \times C^{\infty}$ and has no analytic wave front in the direction of the captive geodesics, then $\underline{u}$ belongs to $F_{\infty}$

Notice that this includes (1.2) because if $u$ is analytic, then $P u$ has no wave front at all!

In the first section, we will prove Theorem 1.1; in the second one, we will show that a little improvement of this proof lets us prove Theorem 1.3. In the last section, we will prove Theorem 1.2 and give an explicit value for the constant $c$.

\section{Proof of Theorem 1.1}

In order to prove this theorem, we will study the following observation problem, that is adjoint to (1.1) 


$$
\mid \begin{aligned}
& \square u=0 \text { over } C \times \mathbb{R}_{t} \\
& \left.u\right|_{\partial C \times \mathbb{R}_{t}}=0 \\
& \left.\left(u, \partial_{t} u\right)\right|_{t=0}=\underline{u} \in E_{0}
\end{aligned}
$$

Let us denote

$$
K \underline{u}=\left.\frac{\partial u}{\partial n}\right|_{\Gamma \times \mathbb{R}_{t}} .
$$

We will use the HUM method to turn observation estimates for problem (2.1) into control properties for problem (1.1).

In problem (2.1), we can separate the variables $\theta$ and $z$, by using Fourier series. Let us give a few definitions with this respect

Definition 2.1. Let $\underline{u}=\left(\sum_{\substack{n \in \mathbb{Z} \\ k \in \mathbb{N}^{*}}} \alpha_{n, k}^{j} \sin k z e^{i n \theta}\right)_{\substack{j=1,2\\}}$ be an initial data in $E_{0}$. We shall denote

$$
\begin{aligned}
& \underline{u} \in E_{0}^{n} \quad \text { if } \quad m \neq n \Rightarrow \alpha_{m, k}^{j}=0, \\
& \underline{u} \in E_{0}^{(1)} \quad \text { if } \quad|k|>|n| \Rightarrow \alpha_{n, k}^{j}=0, \\
& \underline{u} \in E_{0}^{(2)} \quad \text { if } \quad|k| \leq|n| \Rightarrow \alpha_{n, k}^{j}=0, \\
& \underline{u} \in E_{0}^{i, n} \Leftrightarrow \underline{u} \in E_{0}^{(i)} \cap E_{0}^{n} .
\end{aligned}
$$

The space $E_{0}$ can be split into $\left\{\begin{array}{l}E_{0}=\left(\bigoplus_{n} E_{0}^{1, n}\right) \bigoplus\left(\bigoplus_{n} E_{0}^{2, n}\right) \\ \underline{u}=\underline{u}^{1}+\underline{u}^{2}=\sum_{n}\left(u^{1, n}+u^{2, n}\right)\end{array}\right.$

We will call $\underline{u}^{1}$ the "low frequency term" and $\underline{u}^{2}$ the "high frequency term".

These two sequences of vectors form an Hilbert basis for $E_{0}$

$$
e_{n, k}^{1}=\left(\sqrt{\frac{2}{\pi}} \frac{\sin k z e^{i n \theta}}{\sqrt{1+k^{2}+n^{2}}}, 0\right) ; e_{n, k}^{2}=\left(0, \sqrt{\frac{2}{\pi}} \sin k z e^{i n \theta}\right) .
$$

For any $n$, we will restrict the initial data to functions of $E_{0}^{n}$ and estimate the constants that appears in the usual observation inequalities

$$
\|u\|_{E_{0}}^{2} \leq C(n, T)\|K u\|_{L^{2}(\Gamma \times(0, T))}^{2},
$$

(see for instance [4]). As the Bardos-Lebeau-Rauch geometric control hypothesis does not hold, we do not expect these constants $C(n, T)$ to be bounded in $n$. We will see that the way they go to the infinity is closely related to the size of the space of controlled data, through the HUM method.

The estimates will have to take into account both the high frequency and the low frequency term. For the former, the eigenfrequencies are well separated, and a usual Ingham technique will provide us the required estimate. The other term is more complicated, as the gap between eigenfrequencies goes to zero when $n$ goes to the infinity. This part of the spectrum will require a more sophisticated proof, based upon the technique of biorthogonal sequences.

At first, we will give the estimates and show how we can prove the theorem out of them. Then, we will prove those estimates. 
Here is the main proposition upon which our proof will be based.

Proposition 2.2. (low frequency estimate). For any positive $\epsilon$ and $\delta$, there exist a time $T_{1}(\epsilon, \delta)$ smaller than $\frac{C_{\delta}}{\epsilon^{1+\delta}}$ and a positive constant $C_{\epsilon, \delta}$ such that for any integer $n$ and any initial condition $\underline{u}$ in $E_{0}^{n}$, the solution $u$ of problem (2.1) satisfies

$$
\left\|\underline{u}^{1}\right\|_{E_{0}}^{2} \leq C_{\epsilon, \delta} e^{2 \epsilon|n|} \int_{\mathbb{S}^{1}} \int_{-T_{1}(\epsilon, \delta)}^{T_{1}(\epsilon, \delta)}|K \underline{u}(\theta, t)|^{2} d t d \theta .
$$

We will show this proposition later on. Let us first see how we can prove Theorem 1.1 out of them. To do this we will need a few more ingredients. First, a little lemma that we can show right now

Lemma 2.3. (High frequency estimate). For any time $T>2 \sqrt{2}$, there is a constant $C_{T}$ such that for any integer $n$ and any initial condition $\underline{u}$ in $E_{0}^{2, n}$, the solution $u$ of problem (2.1) satisfies

$$
\|\underline{u}\|_{E_{0}}^{2} \leq C_{T}\|K \underline{u}\|_{L^{2}(\Gamma \times(0, T))}^{2} .
$$

Proof. It is a mere application of the classical Ingham technique. As $\underline{u}$ belongs to $E_{0}^{(2)}, u(z, \theta, t)$ can be written as

$$
u(z, \theta, t)=\sum_{k>|n|} \alpha_{n, k}^{ \pm} \sin k z e^{i n \theta} e^{ \pm i t \sqrt{n^{2}+k^{2}}} .
$$

Now, as $T>2 \pi \sqrt{2}>\frac{2 \pi}{\inf _{k>|n|}\left(\sqrt{n^{2}+(k+1)^{2}}-\sqrt{n^{2}+k^{2}}\right)}$,

$$
\int_{\mathbb{S}^{1}} \int_{0}^{T}\left|\frac{\partial u}{\partial z}(z=0, \theta, t)\right|^{2} d t d \theta=4 \pi^{2} \int_{0}^{T}\left|\sum_{k>|n|} k \alpha_{n, k}^{ \pm} e^{ \pm i t \sqrt{n^{2}+k^{2}}}\right|^{2} d t, \geq C \sum_{k>|n|}\left|k \alpha_{n, k}^{ \pm}\right|^{2},
$$

(see [8] p. 222 for a detailed proof of the Ingham estimate for series with gaps).

So

$$
\int_{\mathbb{S}^{1}} \int_{0}^{T}\left|\frac{\partial u}{\partial z}(z=0, \theta, t)\right|^{2} d t d \theta \geq C \sum_{k>|n|}\left|(1+|n|+k) \alpha_{n, k}^{ \pm}\right|^{2} \geq C|| \underline{u} \|_{E_{0}}^{2} .
$$

Hence,

$$
\|K \underline{u}\|_{L^{2}(\Gamma \times(0, T))}^{2} \geq C\|\underline{u}\|_{E_{0}}^{2} .
$$

We will also need the following caracterisation of $F_{T}$ by the HUM method

Lemma 2.4. An initial data $v$ of $E_{-1}$ belongs to $F_{T}$ if and only if there exists a constant $C_{v}$ such that for any initial data $\underline{u}$ in $E_{0}$, the solution $u$ of problem (2.1) satisfies

$$
\left|\langle v, \underline{u}\rangle_{E_{-1}, E_{0}}\right| \leq\left. C_{v}|| K \underline{u}\right|_{L^{2}(\Gamma \times(0, T))} .
$$

The proof of this lemma can be found in [6] or [4].

Now let us prove the theorem. Pick a positive value for $\delta$ and $\epsilon$, and take $v$ in $G_{\epsilon}$. We can put

$$
v(z, \theta)=\sum_{n} e^{-\epsilon|n|} V^{n}(z) e^{i n \theta},
$$

with $\left(\left\|V^{n}(z) e^{i n \theta}\right\|_{E_{-1}}\right)_{n} \in l^{2}(\mathbb{Z})$.

Take $T(\epsilon, \delta)=\sup \left(T_{1}(\epsilon, \delta), 2 \pi \sqrt{2}\right)$. As $T_{1}(\epsilon, \delta) \leq \frac{C}{\epsilon^{1+\delta}}$, for small $\epsilon$, we also have $T(\epsilon, \delta) \leq \frac{C}{\epsilon^{1+\delta}}$. 
For any $\underline{u}$ of $E_{0}$, we have

$$
\left|\langle v, \underline{u}\rangle_{E_{-1}, E_{0}}\right|=\left|\sum_{n} e^{-\epsilon|n|}\left\langle V^{n}(z) e^{i n \theta}, u^{n}\right\rangle_{E_{-1}, E_{0}}\right|
$$

So

$$
\left|\langle v, \underline{u}\rangle_{E_{-1}, E_{0}}\right| \leq \sum_{n} e^{-\epsilon|n|}\left\|V^{n}(z) e^{i n \theta}\right\| E_{E_{-1}}\left\|u^{n}\right\|_{E_{0}} .
$$

Now for any integer $n$ and any $\underline{u}$ in $E_{0}^{n}$, we have

$$
\|\underline{u}\|_{E_{0}}^{2}=\left\|\underline{u}^{1}\right\|_{E_{0}}^{2}+\left\|\underline{u}^{2}\right\|_{E_{0}}^{2} .
$$

So, through Proposition 2.2 and Lemma 2.3,

$$
\|\left.\underline{u}\right|_{E_{0}} ^{2} \leq C_{\epsilon, \delta} e^{2 \epsilon|n|} \int_{\mathbb{S}^{1}} \int_{-T_{1}(\epsilon, \delta)}^{T_{1}(\epsilon, \delta)}|K \underline{u}(\theta, t)|^{2} d t d \theta+C \int_{\mathbb{S}^{1}} \int_{0}^{T(\epsilon, \delta)}\left|K \underline{u}^{2}(\theta, t)\right|^{2} d t d \theta .
$$

So

$$
\begin{array}{r}
\|\left.\underline{u}\right|_{E_{0}} ^{2} \leq \int_{\mathbb{S}^{1}} C_{\epsilon, \delta}^{\prime} e^{2 \epsilon|n|} \int_{-T(\epsilon, \delta)}^{T(\epsilon, \delta)}|K \underline{u}(t)|^{2} d t d \theta+C \int_{\mathbb{S}^{1}} \int_{0}^{T(\epsilon, \delta)}|K \underline{u}(\theta, t)|^{2} d t d \theta \\
+C \int_{\mathbb{S}^{1}} \int_{0}^{T(\epsilon, \delta)}\left|K \underline{u}^{1}(\theta, t)\right|^{2} d t d \theta .
\end{array}
$$

As the problem is well posed,

$$
\|\underline{u}\|_{E_{0}}^{2} \leq \int_{\mathbb{S}^{1}} C_{\epsilon, \delta}^{\prime} e^{2 \epsilon|n|} \int_{-T(\epsilon, \delta)}^{T(\epsilon, \delta)}|K \underline{u}(t)|^{2} d t d \theta+C^{\prime}\left\|\underline{u}^{1}\right\|_{E_{0}}^{2} .
$$

So by Proposition 2.2,

$$
\|\underline{u}\|_{E_{0}}^{2} \leq \int_{\mathbb{S}^{1}} C_{\epsilon, \delta}^{\prime} e^{2 \epsilon|n|} \int_{-T(\epsilon, \delta)}^{T(\epsilon, \delta)}|K \underline{u}(t)|^{2} d t d \theta+C^{\prime} C_{\epsilon, \delta} e^{2 \epsilon|n|} \int_{\mathbb{S}^{1}} \int_{-T_{1}(\epsilon, \delta)}^{T_{1}(\epsilon, \delta)}|K \underline{u}(\theta, t)|^{2} d t d \theta .
$$

Thus

$$
\|\underline{u}\|_{E_{0}}^{2} \leq \int_{\mathbb{S}^{1}} C_{\epsilon, \delta}^{\prime} e^{2 \epsilon|n|} \int_{-T(\epsilon, \delta)}^{T(\epsilon, \delta)}|K \underline{u}(t)|^{2} d t d \theta .
$$

If we put this into $(2.2)$, we get

$$
\begin{aligned}
\left|\left\langle v, \underline{u}^{n}\right\rangle_{E_{-1}, E_{0}}\right| & \leq C_{\epsilon, \delta} \sum_{n}\left\|V^{n}(z) e^{i n \theta}\right\| E_{-1} \sqrt{\int_{\mathbb{S}^{1}} \int_{0}^{2 T(\epsilon, \delta)}\left|K \underline{u}^{n}(\theta, t)\right|^{2} d t d \theta} \\
& \leq C_{\epsilon, \delta} \sqrt{\sum_{n}\left\|V^{n}(z) e^{i n \theta}\right\|_{E_{-1}}^{2}} \sqrt{\sum_{n} \int_{\mathbb{S}^{1}} \int_{0}^{2 T(\epsilon, \delta)}\left|K \underline{u}^{n}(\theta, t)\right|^{2} d t d \theta} \\
& \leq C_{\epsilon, \delta} C_{v}\|K \underline{u}\|_{L^{2}(\Gamma \times(0,2 T(\epsilon, \delta)))}
\end{aligned}
$$

So by Lemma 2.4, $v$ belongs to $F_{T}$. This proves Theorem 1.1. 


\subsection{Proof of Proposition 2.2 (low frequencies)}

As the gap between frequencies goes to zero in the low part of the spectrum, Ingham techniques cannot be used in the proof of this proposition. Instead, we will use a biorthogonal sequence method. The idea is to build a sequence of compactly supported functions $h^{k, n}(t)$ such that for any $j \neq k, h^{k, n}(t)$ is orthogonal to the eigenfunction $e^{t \sqrt{n^{2}+j^{2}}}$, and whose scalar product with $e^{t \sqrt{n^{2}+k^{2}}}$, on the contrary, is not too small. The sequence of functions $h^{k, n}(t)$ is said to be biorthogonal to the sequence $e^{t \sqrt{n^{2}+k^{2}}}$. The observation constants will appear to be related to the $L^{2}$ norms of the functions $h^{k, n}(t)$. We will be able to decrease these norms if we allow the support of the functions to grow.

Let us state precisely the lemma we will prove about the biorthogonal sequence

Lemma 2.5. For any odd integer $q$ and any positive real number $\epsilon$, there is a time $T_{1}(q, \epsilon)$ smaller than $C_{q} \epsilon^{\frac{q+1}{1-q}}$ such that for any couple $\left(n, k_{0}\right)$ in $\mathbb{N}^{* 2}$, we can find an $L^{2}$ function $h_{\epsilon, q}^{k_{0}, n}$ for which the following properties hold

(i) $h_{\epsilon, q}^{k_{0}, n}$ is supported by $\left[-T_{1}(q, \epsilon), T_{1}(q, \epsilon)\right]$.

(ii) $\left\|h_{\epsilon, q}^{k_{0}, n}\right\|_{L^{2}}^{2} \leq C e^{2 \epsilon n}$.

(iii) If $k \neq k_{0}, \quad \int h_{\epsilon, q}^{k_{0}, n}(t) e^{ \pm i t \sqrt{n^{2}+k^{2}}} d t=0$.

(iv) If $\left(n, k_{0}\right) \in I=\left\{(k, n) \in \mathbb{N}^{* 2} \mid k \leq n\right\},\left|\int h_{\epsilon, q}^{k_{0}, n}(t) e^{ \pm i t \sqrt{n^{2}+k_{0}^{2}}} d t\right| \geq \frac{c}{n^{N_{q}}}$.

The constants depend only on $\epsilon$ and $q$.

Moreover, $h_{\epsilon, q}^{k_{0}, n}$ can be chosen as even or odd. They will be denoted $h_{\epsilon_{\epsilon, q}}^{k_{0}, n}$ and $h_{o \epsilon, q}^{k_{0}, n}$.

The reader may keep in mind that $q$ is a technical parameter, designed to get better polynomial estimates for the time T. Let us first show how Proposition 2.2 can be proved out of this lemma.

Let $\underline{u}$ be an element of $E_{0}^{n}$, and put $\underline{u}=\sum_{j}\left(\alpha_{n, j}^{1} e_{n, j}^{1}+\alpha_{n, j}^{2} e_{n, j}^{2}\right)$.

For any $\left(n, k_{0}\right)$ in $I$, any integer $M$ and any $\theta$ in $\mathbb{S}^{1}$,

$$
\begin{aligned}
\int h_{e_{\epsilon, q}}^{k_{0}, n}(t) K & {\left[\sum_{j=1}^{M}\left(\alpha_{n, j}^{1} e_{n, j}^{1}+\alpha_{n, j}^{2} e_{n, j}^{2}\right)\right](t, \theta) d t=\int h_{e_{\epsilon, q}}^{k_{0}, n}(t) \sum_{j=1}^{M}\left[\alpha_{n, j}^{1} K e_{n, j}^{1}(t, \theta)+\alpha_{n, j}^{2} K e_{n, j}^{2}(t, \theta)\right] d t } \\
= & \sum_{j=1}^{M}\left[\alpha_{n, j}^{1} \int h_{\epsilon \in, q}^{k_{0}, n}(t) K e_{n, j}^{1}(t, \theta) d t+\alpha_{n, j}^{2} \int h_{e \epsilon, q}^{k_{0}, n}(t) K e_{n, j}^{2}(t, \theta) d t\right] \\
= & \frac{1}{\sqrt{2 \pi}} e^{i n \theta} \sum_{j=1}^{M}\left[\alpha_{n, j}^{1} \frac{j}{\sqrt{1+j^{2}+n^{2}}} \int h_{e_{\epsilon, q}}^{k_{0}, n}(t)\left(e^{i t \sqrt{j^{2}+n^{2}}}+e^{-i t \sqrt{j^{2}+n^{2}}}\right) d t\right. \\
& \left.+\alpha_{n, j}^{2} \frac{j}{i \sqrt{n^{2}+j^{2}}} \int h_{\epsilon \in, q}^{k_{0}, n}(t)\left(e^{i t \sqrt{j^{2}+n^{2}}}-e^{-i t \sqrt{j^{2}+n^{2}}}\right) d t\right] .
\end{aligned}
$$

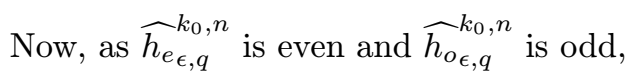

$$
\int h_{e_{\epsilon, q}}^{k_{0}, n}(t) K\left[\sum_{j=1}^{M}\left(\alpha_{n, j}^{1} e_{n, j}^{1}+\alpha_{n, j}^{2} e_{n, j}^{2}\right)\right](t, \theta) d t=\sqrt{\frac{2}{\pi}} e^{i n \theta} \sum_{j=1}^{M} \alpha_{n, j}^{1} \frac{j}{\sqrt{1+j^{2}+n^{2}}} \int h_{e_{\epsilon, q}}^{k_{0}, n}(t) e^{i t \sqrt{j^{2}+n^{2}}} d t .
$$


So by (iii), if $M \geq k_{0}$,

$$
\int h_{e \epsilon, q}^{k_{0}, n}(t) K\left[\sum_{j=1}^{M}\left(\alpha_{n, j}^{1} e_{n, j}^{1}+\alpha_{n, j}^{2} e_{n, j}^{2}\right)\right](t, \theta) d t=\sqrt{\frac{2}{\pi}} e^{i n \theta} \alpha_{n, k_{0}}^{1} \frac{k_{0}}{1+k_{0}^{2}+n^{2}} \int h_{e \epsilon, q}^{k_{0}, n}(t) e^{i t \sqrt{k_{0}^{2}+n^{2}}} d t .
$$

So as $\left(n, k_{0}\right) \in I$, by (iv),

$$
\left|\int h_{e \epsilon, q}^{k_{0}, n}(t) K\left[\sum_{j=1}^{M}\left(\alpha_{n, j}^{1} e_{n, j}^{1}+\alpha_{n, j}^{2} e_{n, j}^{2}\right)\right](t, \theta) d t\right| \geq\left|\alpha_{n, k_{0}}^{1}\right| \frac{c}{n^{N_{q}}}
$$

Therefore, if $M$ goes to the infinity,

$$
\left|\int h_{e_{\epsilon, q}}^{k_{0}, n}(t) K \underline{u}(t, \theta) d t\right| \geq\left|\alpha_{n, k_{0}}^{1}\right| \frac{c}{n^{N_{q}}} .
$$

Similarly

Now

$$
\left|\int h_{o \epsilon, q}^{k_{0}, n}(t) K \underline{u}(t, \theta) d t\right| \geq\left|\alpha_{n, k_{0}}^{2}\right| \cdot \frac{c}{n^{N_{q}}} .
$$

$$
\|\left.\underline{u}^{1}\right|_{E_{0}} ^{2}=\sum_{k \leq n}\left|\alpha_{n, k}^{1}\right|^{2}+\left|\alpha_{n, k}^{2}\right|^{2} .
$$

So by (2.3), for any $\theta \in \mathbb{S}^{1}$,

$$
\left\|\underline{u}^{1}\right\|_{E_{0}}^{2} \leq C \sum_{k \leq n} n^{2 N_{q}}\left|\int h_{e_{\epsilon, q}}^{k_{0}, n}(t) K \underline{u}(t, \theta) d t\right|^{2}+\text { same with } h_{o} .
$$

So by (i),

So by (iii),

$$
\left\|\underline{u}^{1}\right\|_{E_{0}}^{2} \leq C \sum_{k \leq n} n^{2 N_{q}} \int\left|h_{\epsilon, q}^{k_{0}, n}(t)\right|^{2} d t \int_{-T_{1}(q, \epsilon)}^{T_{1}(q, \epsilon)}|K \underline{u}(t, \theta)|^{2} d t
$$

$$
\left\|\underline{u}^{1}\right\|_{E_{0}}^{2} \leq C \sum_{k \leq n} n^{2 N_{q}} e^{2 \epsilon n} \int_{-T_{1}(q, \epsilon)}^{T_{1}(q, \epsilon)}|K \underline{u}(t, \theta)|^{2} d t
$$

Thus

$$
\left\|\underline{u}^{1}\right\|_{E_{0}}^{2} \leq C n^{2 N_{q}^{\prime}} e^{2 \epsilon n} \int_{\mathbb{S}^{1}} \int_{-T_{1}(q, \epsilon)}^{T_{1}(q, \epsilon)}|K \underline{u}(t, \theta)|^{2} d t d \theta,
$$

with $T_{1}(q, \epsilon) \leq C_{q} \frac{q+1}{1^{\frac{q+1}{-q}}}=\frac{C_{\delta}}{\epsilon^{1+\delta}}$ and $\delta \rightarrow 0^{+}$when $q \rightarrow+\infty$.

As we can shift slightly $\epsilon$ to eliminate the polynomial in $n$, we have proved Proposition 2.2.

We still have to prove Lemma 2.5. We will do this in three steps. At first, we build a sequence of functions $f^{k, n}$ for which (i), (iii) and (iv) hold. This construction is explicit, and it is equivalent with the usual way of building biorthogonal sequences, by taking infinite products. The problem with these functions is that their $L^{2}$ norm behaves like $e^{n \pi}$, which is much too big for (ii). Though, we will see that, on the Fourier side, most of this norm is concentrated in $[-n, n]$.

Then, by the stationary phase formula, we will build a sequence of functions $g^{n}$ that are exponentially small on $[-n, n]$ (in frequency), and reasonably bounded outside.

At last, we will put $h=f * g$ and prove how the properties of each functions compensate in such a way that $h$ satisfies (i) to (iv). 


\subsubsection{Definition of $f$ and $g$}

Let us define the sequence of functions $f^{k, n}$.

For any $(n, k)$ in $\mathbb{Z}^{*} \times \mathbb{N}^{*}$ we put

$$
f^{k, n}(t)=F^{-1}\left(\frac{\sin \pi \sqrt{\tau^{2}-n^{2}}}{\sqrt{\tau^{2}-n^{2}}} \frac{1}{\tau^{2}-\left(n^{2}+k^{2}\right)}\right) .
$$

$\left(F^{-1}\right.$ is the reciprocal of the Fourier transform.)

The following properties hold for $f^{k, n}$ :

(f-i) $\widehat{f}^{k, n} \in \mathcal{O}(\mathbb{C}), \widehat{f}^{k, n} \in L^{2}(\mathbb{R})$ and $\forall \tau \in \mathbb{C},\left|\widehat{f}^{k, n}(\tau)\right| \leq C_{n, k} e^{\pi|\Im m \tau|}$. So, by Paley-Wiener, $f^{k, n}$ belongs to $L^{2}(-\pi, \pi)$.

(f-iii) $\forall k \in \mathbb{N}^{*} \backslash\left\{k_{0}\right\}, \widehat{f}^{k_{0}, n}\left( \pm \sqrt{n^{2}+k^{2}}\right)=0$.

(f-iv) $\exists N \in \mathbb{N}, \forall(n, k) \in I, \widehat{f}^{k, n}\left( \pm \sqrt{n^{2}+k^{2}}\right) \geq \frac{c}{n^{N}}$

However, $\left\|f^{k, n}\right\|_{L^{2}} \geq C e^{n}$, therefore (ii) doesn't hold. Instead, we have the following estimate for any $\left(n, k_{0}\right)$ in $\mathbb{Z}^{*} \times \mathbb{N}^{*}$ and any $\tau$ in $[-n, n]$

$$
\left|\widehat{f}^{k_{0}, n}(\tau)\right| \leq C e^{n \pi \sqrt{1-\left|\frac{\tau}{n}\right|^{2}}}
$$

For any odd integer $q$, let $h_{q}(x)$ be the solution of $y^{\prime}=1+y^{q-1}$ such that $y(0)=0$. It is defined over $\left(-x_{q}, x_{q}\right)$ for a positive $x_{q}$. It is odd, strictly increasing and analytic. Moreover, $h_{q}(x)=x+\alpha_{q} x^{q}+o\left(x^{q}\right)$ when $x$ is close to 0 , with a positive $\alpha_{q}$ and $h_{q}(x) \rightarrow+\infty$ when $x \rightarrow x_{q}$.

Its reciprocal function will be denoted $H_{q}$. It is defined over $\mathbb{R}$, odd, bounded by $x_{q}$ and satisfies $H_{q}(x)=$ $x-\alpha_{q} x^{q}+o\left(x^{q}\right)$ when $x$ is close to 0 .

Pick $\delta>1$, close to 1 , that will be fixed later. We define the functions $g_{+}$as follows for any odd integer $q$, any integer $n$ and any real time $T$

$$
g_{+T, q}^{n}(t)=\mathbf{1}_{(-T, T)} e^{i n \frac{T}{\delta x_{q}} h_{q}\left(\frac{x_{q}}{T} t\right)} .
$$

So

$$
{\widehat{g_{+}}}_{T, q}^{n}(\tau)=\int_{-T}^{T} e^{i n \frac{T}{\delta x_{q}} h_{q}\left(\frac{x_{q}}{T} t\right)-i \tau t} d t .
$$

Put $\Psi_{q}(s)=\frac{T}{x_{q}} H_{q}\left(\frac{\delta x_{q}}{T} s\right)$, then

$$
{\widehat{g_{+}}}_{T, q}^{n}(\tau)=\int_{-\infty}^{+\infty} e^{i n s-i \tau \Psi_{q}(s)} \Psi_{q}^{\prime}(s) d s .
$$

If we write $\theta_{q}(s)=\frac{1}{x_{q}} H_{q}\left(\delta x_{q} s\right)$, then

$$
\widehat{g_{+T, q}}(\tau)=\int_{-\infty}^{+\infty} \theta_{q}^{\prime}\left(\frac{s}{T}\right) e^{i n T\left(\frac{s}{T}-\frac{\tau}{n} \theta_{q}\left(\frac{s}{T}\right)\right)} d s=T \int_{-\infty}^{+\infty} \theta_{q}^{\prime}(v) e^{i n T\left(v-\frac{\tau}{n} \theta_{q}(v)\right)} d v
$$

We will show later on the following lemma about the functions $g_{+T, q}$. 
Lemma 2.6. For big enough $T$, there are three positive real constants $C_{q}^{1}, C_{q, T}^{2}, c_{q, T, \delta}^{3}$ and an integer $n(q)$ such that

- for any integer $n$ and any real number $\tau$ smaller than $\frac{n}{\delta}$,

$$
\left|\widehat{g}+T, q^{n}(\tau)\right| \leq C_{q, T}^{2} e^{-T n C_{q}^{1} \min \left\{\left(\frac{1}{\delta}-\frac{\tau}{n}\right)^{\frac{q}{q-1}}, 1\right\}} .
$$

- For any integer $n$ greater than $n(q)$ and any integer $k_{0}$ smaller than $n$, there is a time $T_{n, k_{0}}$ in $[T, T+1]$ such that

$$
\left|\widehat{g+}_{+T_{n, k_{0}}, q}^{n}\left(\sqrt{n^{2}+k_{0}^{2}}\right)\right| \geq \frac{c_{q, T, \delta}^{3}}{\sqrt{n}} .
$$

Let us see how this lemma allows us build a sequence of functions $h$ that satisfy Lemma 2.5.

\subsubsection{Definition and properties of $h$}

First, let us notice that we can get a lemma that is symmetrical to Lemma 2.6 for functions $g_{-T, q}^{n}(t)=$ $\mathbf{1}_{(-T, T)} e^{-i n \frac{T}{\delta x_{q}} h_{q}\left(\frac{x_{q}}{T} t\right)}$. $(t$ is replaced by $-t)$.

As $g_{-T, q}^{n}=\overline{g_{+T, q}^{n}}, T_{n, k_{0},+}=T_{n, k_{0},-}$.

So if we put

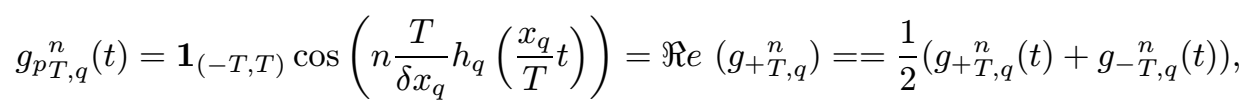

we get, for $\left|\frac{\tau}{n}\right| \leq \frac{1}{\delta}$

$$
\left|\widehat{g}_{p T, q}^{n}(\tau)\right| \leq C_{q, T} e^{-T n C_{q}\left(\frac{1}{\delta}-\left|\frac{\tau}{n}\right|\right)^{\frac{q}{q-1}}}
$$

For $n \geq n(\epsilon)$ and $k_{0} \leq n$, as $C_{q, T}^{2} e^{-n T_{u} C_{q}^{1}} \leq \frac{c_{q, T, \delta}^{3}}{2 \sqrt{n}}$ if $n$ is big enough,

$$
\left|{\widehat{g_{ \pm}}}_{T_{n, k_{0}}, q}^{n}(\tau)\right| \leq \frac{1}{2}\left|{\widehat{g_{\mp} T_{n, k_{0}}, q}}^{n}(\tau)\right| \text { for } \tau=\mp \sqrt{n^{2}+k_{0}^{2}} .
$$

As we can increase the constant to deal with the finite number of $(n, k)$ in $I$ whose $n$ is not big enough, we get for any $\left(n, k_{0}\right)$ in $I$ and $\tau= \pm \sqrt{n^{2}+k_{0}^{2}}$,

$$
\left|\widehat{g}_{T_{n, k_{0}}, q}(\tau)\right| \geq \frac{c_{q, T, \delta}}{\sqrt{n}}
$$

So we get for $g_{p}$ the following lemma, that corresponds to Lemma 2.6

Lemma 2.7. For big enough $T$, there are three positive real constants $C_{q}^{1}, C_{q, T}^{2}, c_{q, T, \delta}^{3}$ and an integer $n(q)$ such that

- for any integer $n$ and any real number $\tau$ in $\left[-\frac{n}{\delta}, \frac{n}{\delta}\right]$,

$$
\left|\widehat{g}_{p, q}^{n}(\tau)\right| \leq C_{q, T}^{2} e^{-T n C_{q}^{1}\left(\frac{1}{\delta}-\left|\frac{\tau}{n}\right|\right)^{\frac{q}{q-1}}} .
$$

- For any integer $n$ greater than $n(q)$ and any integer $k_{0}$ smaller than $n$, there is a time $T_{n, k_{0}}$ in $[T, T+1]$ such that

$$
\left|\widehat{g}_{p_{T_{n, k_{0}}, q}}^{n}\left( \pm \sqrt{n^{2}+k_{0}^{2}}\right)\right| \geq \frac{c_{q, T, \delta}^{3}}{\sqrt{n}} .
$$


We could get similar results for $g_{i T, q}^{n}(t)=\mathbf{1}_{(-T, T)} \sin \left(n \frac{T}{\delta x_{q}} h_{q}\left(\frac{x_{q}}{T} t\right)\right)=\Im m\left(g_{+T, q}\right) \cdot g_{e}$ is even whereas $g_{o}$ is odd.

Now let us define the functions $h$ by a convolution product, as was announced.

Let $\epsilon$ be a positive real number. Choose $\delta_{\epsilon}$ such that $\pi \sqrt{1-\left(\frac{1}{\delta_{\epsilon}}\right)^{2}}=\frac{\epsilon}{2}$ and $T^{\epsilon}$ such that

$$
\sup _{\beta \in\left[0, \frac{1}{\delta_{\epsilon}}\right]}\left(\pi \sqrt{1-\beta^{2}}-C_{q}^{1} T^{\epsilon}\left(\frac{1}{\delta_{\epsilon}}-\beta\right)^{\frac{q}{q-1}}\right) \leq \epsilon .
$$

As the derivative of the function to maximize is $\frac{-\pi \beta}{\sqrt{1-\beta^{2}}}+\frac{q}{q-1} T^{\epsilon} C_{q}^{1}\left(\frac{1}{\delta_{\epsilon}}-\beta\right)^{\frac{1}{q-1}}$, it is enough to choose $T^{\epsilon}$ such that this derivative is 0 for value $\beta_{\epsilon}$ such that $\pi \sqrt{1-\beta_{\epsilon}^{2}}=\epsilon$.

We have $\delta_{\epsilon}=1+\frac{\epsilon^{2}}{8 \pi^{2}}+o\left(\epsilon^{2}\right), \beta_{\epsilon}=1-\frac{\epsilon^{2}}{2 \pi^{2}}+o\left(\epsilon^{2}\right)$. Thus $\frac{1}{\delta_{\epsilon}}-\beta_{\epsilon} \sim c_{q} \epsilon^{2}$, hence

$$
T^{\epsilon} \sim c_{q} \epsilon^{\frac{q+1}{1-q}}
$$

Now, let us define a sequence of times $T_{n, k_{0}}^{\epsilon}$.

For $k_{0} \leq n$, we take the values given by Lemma 2.7 with $T=T^{\epsilon}$.

For $k_{0}>n$, we put $T_{n, k_{0}}^{\epsilon}=T^{\epsilon}$.

Then

$$
T_{n, k_{0}}^{\epsilon} \in\left[T^{\epsilon}, T^{\epsilon}+1\right]
$$

so

$$
c_{q}^{1} \epsilon^{\frac{q+1}{1-q}} \leq T_{n, k_{0}}^{\epsilon} \leq c_{q}^{2} \epsilon^{\frac{q+1}{1-q}} .
$$

Let us define the sequence of functions $h$ as follows

$$
\begin{aligned}
& {\widehat{h_{e}, q}}_{e^{\prime}, n}^{k_{0}}(\tau)=\widehat{f}^{k_{0}, n} \cdot \widehat{g}_{e T_{n, k_{0}}^{\epsilon}, q}^{n}(\tau), \\
& {\widehat{h_{o \epsilon, q}}}_{k_{0}, n}^{k^{\prime}}(\tau)=\widehat{f}^{k_{0}, n} \cdot \widehat{g}_{o T_{n, k_{0}}^{\epsilon}, q}^{n}(\tau) .
\end{aligned}
$$

The index $h_{e}$ or $h_{o}$ means that $h$ is even or odd (This index will not be written for results valid in both cases).

Now let us check each of the properties of Lemma 2.5 for the functions $h$.

- (i) This point is easy because $h_{\epsilon, q}^{k_{0}, n}$ is the convolution product of $f^{k_{0}, n}$ which is supported by $[-\pi, \pi]$ and $g_{T_{n, k_{0}}^{\epsilon}, q}^{n}$, which is supported by $\left[-T_{n, k_{0}}^{\epsilon}, T_{n, k_{0}}^{\epsilon}\right]$.

So if we put $T_{1}(q, \epsilon)=\pi+T_{n, k_{0}}^{\epsilon}, h_{\epsilon, q}^{k_{0}, n}$ is supported by $\left[-T_{1}(q, \epsilon), T_{1}(q, \epsilon)\right]$ with $T_{1}(q, \epsilon) \leq C_{q} \epsilon^{\frac{q+1}{1-q}}$.

- (ii) This is where the small values of $g$ compensate the big size of $f$. As over $\mathbb{R} \backslash[-n, n]$ the $L^{2}$ norm of $\widehat{f}$ is bounded by a polynomial and $|\widehat{g}|_{L^{\infty}}$ is bounded by $2 T_{1}(q, \epsilon)$, the problem is concentrated in $[-n, n]$.

We must estimate $\int_{-n}^{n}\left|\widehat{h}_{\epsilon, q}^{k_{0}, n}(\tau)\right|^{2} d \tau$.

Now over $\frac{\tau}{n} \in[-1,1]$, by $(2.4)$,

So if $\left|\frac{\tau}{n}\right| \geq \frac{1}{\delta_{\epsilon}}$ then

$$
\left|\widehat{f}^{k_{0}, n}(\tau)\right|^{2} \leq C e^{2 \pi n \sqrt{1-\left|\frac{\tau}{n}\right|^{2}}}
$$

$$
\left|\widehat{h}_{\epsilon, q}^{k_{0}, n}(\tau)\right|^{2} \leq C e^{\epsilon n}
$$


Now by Lemma 2.7 , for $\left|\frac{\tau}{n}\right| \leq \frac{1}{\delta_{\epsilon}}$,

$$
\left|\widehat{g}_{T_{n, k_{0}}^{\epsilon}, q}^{n}\right|^{2} \leq C e^{-2 T_{n, k_{0}}^{\epsilon} n C_{q}^{1}\left(\frac{1}{\delta_{\epsilon}}-\left|\frac{\tau}{n}\right|\right)^{\frac{q}{q-1}}} .
$$

So from (2.7),

$$
\left|\widehat{h}_{\epsilon, q}^{k_{0}, n}(\tau)\right|^{2} \leq C e^{2 \epsilon n}
$$

Hence

$$
\left\|\widehat{h}_{\epsilon, q}^{k_{0}, n}\right\|_{L^{2}}^{2} \leq C e^{2 \epsilon n}
$$

- (iii) This point is a direct consequence of property (f-iii). Indeed if $k \neq k_{0}, \widehat{f}^{k_{0}, n}\left( \pm \sqrt{n^{2}+k^{2}}\right)=0$, so $\widehat{h}_{\epsilon, q}^{k_{0}, n}\left( \pm \sqrt{n^{2}+k^{2}}\right)=0$. Which is exactly (iii) on the Fourier side.

- (iv) This is true because (f-iv) is not worsened too much by the product with $g$.

Indeed for $\left(n, k_{0}\right) \in I$, by (f-iv) and (2.6),

$$
\left|\widehat{h}_{\epsilon, q}^{k_{0}, n}\left( \pm \sqrt{n^{2}+k_{0}^{2}}\right)\right| \geq \frac{C}{n^{N}} \frac{c_{q, T_{\epsilon}, \delta_{\epsilon}}}{\sqrt{n}} \geq \frac{C_{q, \epsilon}}{n^{N^{\prime}}} .
$$

Which is again the Fourier transcription of (iv).

In order to end our proof, we only have to prove Lemma 2.6 left.

\subsubsection{Proof of Lemma 2.6}

Recall that

$$
{\widehat{g_{+}}}^{n}(\tau)=T \int_{-\infty}^{+\infty} \theta_{q}^{\prime}(v) e^{i n T\left(v-\frac{\tau}{n} \theta_{q}(v)\right)} d v .
$$

Put $\alpha=n T$ and $\beta=\frac{\tau}{n}$.

We will consider

$$
\phi(\alpha, \beta)=\int \theta_{q}^{\prime}(v) e^{i \alpha\left[v-\beta \theta_{q}(v)\right]} d v
$$

with $\alpha$ going to $+\infty$.

Depending upon the value of $\beta$ as compared with $\frac{1}{\delta}$, the phase in the integral will have stationary points or not. This will allow us to use classical asymptotic estimates in both cases.

Case $\beta \leq \frac{1}{\delta}$

In that case, the phase is not stationary. There will be an exponential decrease with respect to $\alpha$. Let us prove it by shifting slightly in the imaginary direction. 


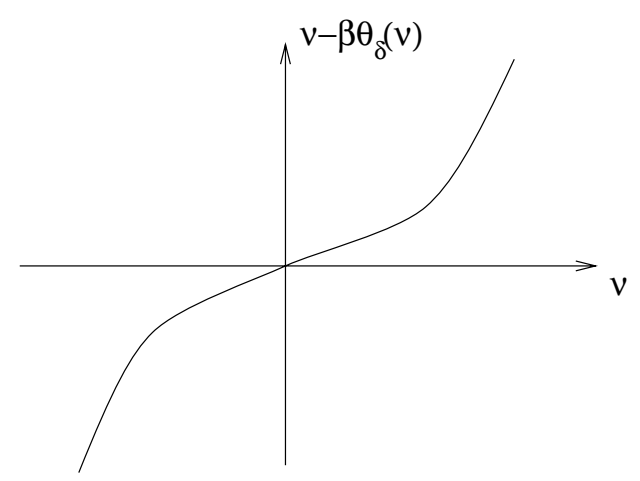

Figure 1. Non stationnary phase.

For any real number $v$ and any $\beta$ smaller than $\frac{1}{\delta}$,

$$
\begin{aligned}
\Im m\left(v+i \epsilon-\beta \theta_{q}(v+i \epsilon)\right) & =\epsilon-\beta \Im m \theta_{q}(v+i \epsilon) \\
& =\epsilon-\beta \Im m\left(\theta_{q}(v+i \epsilon)-\theta_{q}(v)\right) \\
& =\epsilon-\beta \Im m \int_{v}^{v+i \epsilon} \theta_{q}^{\prime}(z) d z \\
& =\epsilon-\beta \Im m \int_{v}^{v+i \epsilon} \frac{\delta d z}{1+\delta^{q-1} x_{q}^{q-1} z^{q-1}} \\
& =\epsilon-\beta \epsilon \delta \Re \int_{0}^{1} \frac{d u}{1+\delta^{q-1} x_{q}^{q-1}(v+i \epsilon u)^{q-1}}
\end{aligned}
$$

Therefore, if $\beta \leq 0$,

$$
\Im m\left(v+i \epsilon-\beta \theta_{q}(v+i \epsilon)\right) \geq \epsilon \text { if } \beta \leq 0 .
$$

If $\beta>0$, then

$$
\Im m\left(v+i \epsilon-\beta \theta_{q}(v+i \epsilon)\right) \geq \epsilon-\beta \epsilon \delta\left|\int_{0}^{1} \frac{d u}{1+\delta^{q-1} x_{q}^{q-1}(v+i \epsilon u)^{q-1}}\right| .
$$

Now for any real number $v$,

$$
\underbrace{\left|\int_{0}^{1} \frac{d u}{1+\delta^{q-1} x_{q}^{q-1}(v+i \epsilon u)^{q-1}}\right|}_{I} \leq \frac{1}{1-c_{q} \epsilon^{q-1}}
$$

because

either $v \gg \epsilon$, then $I \leq \frac{c}{1+v^{q-1}} \leq 1$, either $v \leq M_{q} \epsilon$ and in that case $|v+i \epsilon u|^{q-1} \leq C_{q} \epsilon^{q-1} \Rightarrow$ $\Rightarrow\left|1+\delta^{q-1} x_{q}^{q-1}(v+i \epsilon u)^{q-1}\right| \geq 1-c_{q} \epsilon^{q-1} \Rightarrow I \leq \frac{1}{1-c_{q} \epsilon^{q-1}}$. 
Thus

$$
\begin{aligned}
\Im m\left(v+i \epsilon-\beta \theta_{q}(v+i \epsilon)\right) & \geq \epsilon-\frac{\beta \delta \epsilon}{1-c_{q} \epsilon^{q-1}} \\
& \geq \epsilon(1-\delta \beta)-c_{q}^{\prime} \beta \epsilon^{q} \\
& \geq \epsilon\left(\frac{1}{\delta}-\beta\right)-c_{q}^{\prime} \beta \epsilon^{q}
\end{aligned}
$$

Now

$$
\min _{\epsilon} \epsilon\left(\frac{1}{\delta}-\beta\right)-c_{q} \beta \epsilon^{q}=c_{q}^{\prime}\left(\frac{1}{\delta}-\beta\right)^{\frac{q}{q-1}} \beta^{\frac{1}{1-q}} \geq c_{q}^{\prime \prime}\left(\frac{1}{\delta}-\beta\right)^{\frac{q}{q-1}} .
$$

We can pick two very small real numbers $\epsilon$ and $c_{q}$ such that for any real number $v$,

$$
\left\{\begin{array}{l}
\left.\left.\Im m\left(v+i \epsilon-\beta \theta_{q}(v+i \epsilon)\right) \geq c_{q}^{t e}\left(\frac{1}{\delta}-\beta\right)^{\frac{q}{q-1}} \text { if } \beta \in\right] 0, \frac{1}{\delta}\right] \\
\Im m\left(v+i \epsilon-\beta \theta_{q}(v+i \epsilon)\right) \geq c_{q}^{t e} \text { if } \beta \leq 0 .
\end{array}\right.
$$

So if we shift the integration contour for $\phi$ can be shifted from $\mathbb{R}$ to $\mathbb{R}+i \epsilon$, we get

$$
\phi(\alpha, \beta)=\int \theta_{q}^{\prime}(v+i \epsilon) e^{i \alpha\left[v+i \epsilon-\beta \theta_{q}(v+i \epsilon)\right]} d v .
$$

Finally, as $\theta_{q}^{\prime}(v+i \epsilon)=\frac{\delta}{1+\left(\delta x_{q}(v+i \epsilon)\right)^{q}-1}$, we have $\left|\theta_{q}^{\prime}(v+i \epsilon)\right| \leq \frac{C_{q}}{1+v^{q-1}}$.

Hence, for any real number $\alpha$ and any $\beta \leq \frac{1}{\delta}$,

$$
|\phi(\alpha, \beta)| \leq \int \frac{C_{q}}{1+v^{q-1}} e^{-\alpha c_{q} \min \left\{\left(\frac{1}{\delta}-\beta\right)^{\frac{q}{q-1}}, 1\right\}} d v \leq C_{q} e^{-\alpha c_{q} \min \left\{\left(\frac{1}{\delta}-\beta\right)^{\frac{q}{q-1}}, 1\right\}} .
$$

So if we go back to the original notation, for $\frac{\tau}{n} \leq \frac{1}{\delta}$,

$$
\left|\widehat{g}_{+T, q}^{n}(\tau)\right| \leq C_{q} T e^{-n T c_{q} \min \left\{\left(\frac{1}{\delta}-\frac{\tau}{n}\right)^{\frac{q}{q-1}}, 1\right\}} .
$$

Which is the first part of Lemma 2.6.

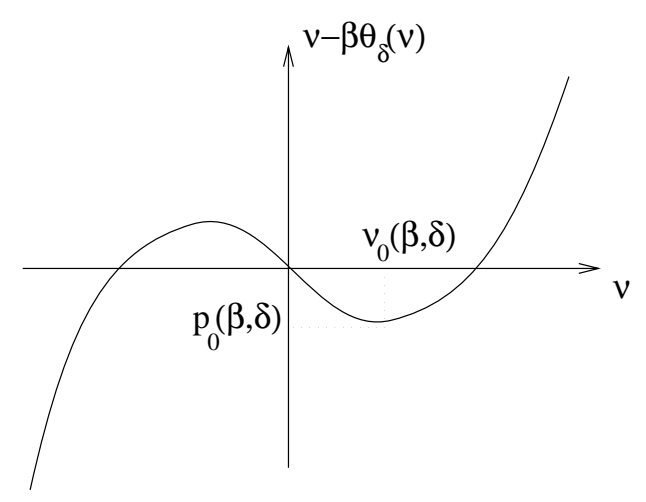

FiguRE 2. Stationnary phase. 
Case $\beta>1$.

In that case, the phase is stationary for two opposite values. We can use the stationary phase formula (see for instance [7] p. 431), and get

$$
\phi(\alpha, \beta)=\left(\frac{1}{\sqrt{H_{\beta, \delta}}} \cos \alpha p_{0}(\beta, \delta)\right)\left[\frac{\theta_{q}^{\prime}\left(v_{0}(\beta, \delta)\right)}{\sqrt{\alpha}}+\sum_{j=1}^{N} \frac{a_{j}(\beta, \delta)}{\alpha^{j} \sqrt{\alpha}}\right]+r_{\beta, \delta}(\alpha),
$$

where $r_{\beta, \delta}(\alpha) \leq \frac{C_{\beta}}{\alpha^{N+1}}$ and $\alpha \geq A_{\beta, \delta} . H_{\beta, \delta}$ denotes the Hessian at the critical points.

Here, $C$ and $A$ are continuous with respect to $\beta$ and $\delta$, and $a_{j}(\beta, \delta)$ depends only on the first $2 j+1$ derivatives of $v \mapsto \theta_{q}(v)$ at $v=v_{0}(\beta, \delta)$ and on $H_{\beta, \delta}$.

Let us compute $p_{0}(\beta, \delta)$.

$$
\begin{aligned}
\frac{\partial}{\partial v}\left(v-\beta \theta_{q}(v)\right)=0 & \Leftrightarrow 1-\frac{\beta \delta}{1+\delta^{q-1} x_{q}^{q-1} v^{q-1}}=0 \\
& \Rightarrow 1+\delta^{q-1} x_{q}^{q-1} v_{0}^{q-1}(\beta, \delta)=\beta \delta \\
& \Rightarrow v_{0}(\beta, \delta)=\frac{1}{\delta x_{q}}(\delta \beta-1)^{\frac{1}{q-1}} .
\end{aligned}
$$

If $\beta$ takes the values $\frac{\sqrt{n^{2}+k^{2}}}{n}$ with $(n, k) \in I$, which implies that $\sqrt{2} \geq \beta \geq 1$, we get

$$
C \geq v_{0}(\beta, \delta),\left|p_{0}(\beta, \delta)\right|,\left|H_{\beta, \delta}\right| \geq c_{\delta}
$$

so

$$
1 \geq \theta_{q}^{\prime}\left(v_{0}(\beta, \delta)\right) \geq c_{q}
$$

Moreover $a_{j}(\beta, \delta) \leq C_{j, \delta}$.

Let $T$ be a positive time. As $\left|p_{0}(\beta, \delta)\right| \geq c_{\delta}$, for any $\left(n, k_{0}\right)$ in $I$, we can pick a time $T_{n, k_{0}}$ in $[T, T+1]$ such that $\cos \left(n T_{n, k_{0}} p_{0}\left(\frac{\sqrt{n^{2}+k_{0}^{2}}}{n}, \delta\right)\right) \geq c_{\delta}^{\prime}$.

So if $T$ is greater than $T_{u}$, for any integer $n \geq n(q)$ if $\alpha=T n$ and $\beta=\frac{\sqrt{n^{2}+k_{0}^{2}}}{n}$,

$$
\begin{gathered}
\left|\frac{\theta_{q}^{\prime}\left(v_{0}(\beta, \delta)\right)}{\sqrt{\alpha}}+\sum_{j=1}^{N} \frac{a_{j}(\beta, \delta)}{\alpha^{j} \sqrt{\alpha}}\right| \geq \frac{\left|\theta_{q}^{\prime}\left(v_{0}(\beta, \delta)\right)\right|}{2 \sqrt{\alpha}}, \\
\left|r_{\beta, \delta}(\alpha)\right| \leq c_{\delta}^{\prime} \frac{\left|H_{\beta, \delta}\right| \theta_{q}^{\prime}\left(v_{0}(\beta, \delta)\right)}{4 \sqrt{\alpha}} .
\end{gathered}
$$

So for any integer $n \geq n(q)$ any time $T>T_{u}$, and any integer $k_{0} \leq n$, we have found a time $T_{n, k_{0}}$ in $[T, T+1]$ such that

$$
\left|\phi\left(n T_{n, k_{0}}, \frac{\sqrt{n^{2}+k_{0}^{2}}}{n}\right)\right| \geq \frac{c_{\delta}^{\prime}|H| \theta_{q}^{\prime}\left(v_{0}\left(\frac{\sqrt{n^{2}+k_{0}^{2}}}{n}, \delta\right)\right)}{4 \sqrt{n} \sqrt{T_{n, k_{0}}}} \geq \frac{c}{\sqrt{n}} .
$$

Which means that

$$
\left|{\widehat{g_{+}}}_{T_{n, k_{0}}, q}^{n}\left(\sqrt{n^{2}+k_{0}^{2}}\right)\right| \geq \frac{C_{T, q, \delta}}{\sqrt{n}} .
$$

This is the second part of Lemma 2.6.

This completes the proof of Theorem 1.1. 


\section{Proof of Theorem 1.3}

In order to prove this theorem, we will use two lemmas. The first one will deal with high frequencies, by proving that the lack of analytic wave front in the required direction implies that the amplitudes corresponding to these frequencies decrease exponentially. The second one will deal with low frequencies, and give an estimate of each of their amplitude. Here are those lemmas

Lemma 3.1. (high frequencies). If an initial condition $v=\left(\sum v_{n, k}^{i} e^{i(k z+n \theta)}\right)_{i=0,1}$ of $E_{0}$, is such that Pv has no analytic wave front in the direction of the captive geodesics, then there are two positive real numbers $\eta$ and e such that

$$
\left(v_{n, k} e^{\epsilon|n|}\right)_{n, k} \in l^{2}\left(\left\{(n, k) \in \mathbb{Z}^{2}|| k|\leq \eta| n \mid\right\}\right)
$$

Lemma 3.2. (low frequencies). There is an integer $N$ such that for any positive $\eta$, there are a positive constant $C(\eta)$ and a positive time $T(\eta)$ ensuring that for any initial condition $\underline{u}=\left(\sum u_{n, k}^{i} e^{i(k z+n \theta)}\right)_{i=0,1}$ in $E_{0}$ and any integers $n$ and $k$ such that $|n| \leq \eta|k|$,

$$
\left|u_{n, k}^{i}\right| \leq C(\eta)(1+|n|+|k|)^{N}|K u|_{L^{2}(\Gamma \times(0, T(\eta)))}
$$

Let us show how we can prove Theorem 1.3 out of these two lemmas. Let $v$ be an initial data in $E_{-1}$ that satisfies the hypotheses of the theorem, and $u$ be any element of $E_{0}$.

$$
|\langle v, u\rangle|=\left|\sum_{n, k}\left(v_{n, k}^{1} u_{n, k}^{0}-v_{n, k}^{0} u_{n, k}^{1}\right)\right| \leq \sum_{n, k}\left|v_{n, k}^{1} u_{n, k}^{0}\right|+\sum_{n, k}\left|v_{n, k}^{0} u_{n, k}^{1}\right|
$$

As $v_{n, k}^{i}$ and $u_{n, k}^{i}$ satisfy the same estimates whether $i=0$ or $i=1$, we shall write $|\langle v, u\rangle| \leq \sum_{n, k}\left|v_{n, k} u_{n, k}\right|$ in order to simplify the notation. Pick the values of $\eta$ and $\epsilon$ given by Lemma 3.1. Note that they depend only on $v$.

$$
|\langle v, u\rangle| \leq \sum_{|k| \leq \eta|n|}\left|v_{n, k} e^{\epsilon|n|} u_{n, k} e^{-\epsilon|n|}\right|+\sum_{|k|>\eta|n|}\left|v_{n, k}(1+|n|+|k|)^{N+2} \frac{u_{n, k}}{(1+|n|+|k|)^{N+2}}\right|
$$

So

$$
\begin{aligned}
|\langle v, u\rangle| \leq & \underbrace{\left(\sum_{\mid k, k}\left|v_{n, k} e^{\epsilon|n|}\right|^{2}\right)^{\frac{1}{2}}}_{|k| \leq \eta|n|}\left(\sum_{|k| \leq \eta|n|}\left|u_{n, k}\right|^{2} e^{-2 \epsilon|n|}\right)^{\frac{1}{2}} \\
& =\underbrace{\left(\sum_{|k|>\eta|n|}\left|v_{n, k}^{2}(1+|n|+|k|)^{2(N+2)}\right|\right)^{\frac{1}{2}}}_{=C_{1}(v)<+\infty \text { by Lemma 3.1 }}\left(\sum_{|k|>\eta|n|} \frac{\left|u_{n, k}\right|^{2}}{(1+|n|+|k|)^{2(N+2)}}\right)^{\frac{1}{2}} .
\end{aligned}
$$


So

$$
\begin{aligned}
|\langle v, u\rangle| & \leq C_{1}(v)\left(\sum_{n}\left|u^{n, 1}\right|_{E_{0}}^{2} e^{-2 \epsilon|n|}\right)^{\frac{1}{2}}+C_{2}(v) \underbrace{|K u|_{L^{2}(\Gamma \times(0, T(\eta))}\left(\sum_{n, k} \frac{1}{(1+|n|+|k|)^{4}}\right)^{\frac{1}{2}}}_{\text {by Lemma 3.2 }} \\
& \leq C_{1}(v) \underbrace{\left(\sum_{n}|K u|_{L^{2}(\Gamma \times(0, T(\epsilon)))}^{2}\right)^{\frac{1}{2}}}_{\text {by proposition } 2.2}+C_{2}(v)|K u|_{L^{2}} \\
& \leq C(v)|K u|_{L^{2}(\Gamma \times(0, T(\epsilon))) .}
\end{aligned}
$$

So by Lemma $2.4, v$ belongs to $F_{T(\epsilon)}$.

\subsection{Proof of Lemma 3.1}

As some readers may not been familiar with the notion of analytic wave front, we shall give a simple definition for this notion. Let us define a kind of FBI transform

Definition 3.3. For any distribution $f$ in $\mathcal{S}^{\prime}\left(\mathbb{R}^{2}\right)$, we denote

$$
B f(z)=\int e^{i z \cdot \xi} \widehat{f}(\xi) e^{-|\xi|} d \xi .
$$

Notice that any distribution is transformed by $B$ into a function that is holomorphic over $\left\{\left(\Im m z_{1}\right)^{2}+\right.$ $\left.\left.\left(\Im m z_{2}\right)^{2}\right\}<1\right\}$.

This transform has been introduced by Lebeau in [5]. Although it is not strictly local, it is very useful when one wants to study different types of wavefront sets of $f$. For instance, the point $(x, \xi)$ does not belong to $W F(f)$ if and only if $B f$ and all its derivatives have a limit at $x-i \frac{\xi}{|\xi|}$.

We shall say, as in [5] that a function has no analytic wave front at the point $(x, \xi)$ if and only if $B f$ can be continued as an holomorphic function over a complex neighborhood of $x-i \frac{\xi}{|\xi|}$. This definition of the analytic wave front set, due to Lebeau, is not the only one. It is not the first one either. However, it is the least technical one and in many cases the more practical to compute.

In the periodic case, $B f$ can be expressed in terms of Fourier coefficients Let $f(z, \theta)=\sum_{n, k} a_{n, k} e^{i n \theta+i k z}$, then

$$
\widehat{f}\left(\xi_{1}, \xi_{2}\right)=\sum_{n, k} a_{n, k} \delta_{\xi_{1}=k} \otimes \delta_{\xi_{2}=n} .
$$

So

$$
\begin{aligned}
B f(z, \theta) & =\int e^{i\left(z \xi_{1}+\theta \xi_{2}\right)} e^{-|\xi|} \widehat{f}\left(\xi_{1}, \xi_{2}\right) d \xi \\
& =\sum_{n, k} \int e^{i\left(z \xi_{1}+\theta \xi_{2}\right)-\sqrt{\xi_{1}^{2}+\xi_{2}^{2}}} a_{n, k} \delta_{\xi_{1}=k} \otimes \delta_{\xi_{2}=n} d \xi_{1} d \xi_{2} \\
& =\sum_{n, k} e^{i n \theta+i k z-\sqrt{n^{2}+k^{2}}} a_{n, k} .
\end{aligned}
$$


Let $v$ satisfy the hypotheses of Lemma 3.1. The lack of analytic wave front of $P v$ means that $B P v$, that is holomorphic over the set $(\Im m \theta)^{2}+(\Im m z)^{2}<1$ has an holomorphic extension in a complex neighborhood of the set $\Im m z=0,|\Im m \theta|=1$. Therefore there is a positive real number $\epsilon$ such that $B P v$ is analytic over $\Im m z=0, \Im m \theta= \pm(1+2 \epsilon)$. Therefore, the periodic functions that maps $(z, \theta) \in \mathbb{R}^{2}$ to $B P v(z, \theta \pm i(1+2 \epsilon))$ are analytic. They belong to $L^{2}\left([0,2 \pi]^{2}\right)$. So the sequence of their Fourier coefficients belong to $l^{2}\left(\mathbb{Z}^{2}\right)$.

Now as $B P v$ is an holomorphic function, by shifting the integration contour in $\theta$ from $[0,2 \pi]$ to $\pm i(1+2 \epsilon)+$ $[0,2 \pi]$, we know that these coefficient are equal to $e^{ \pm(1+2 \epsilon) n}$ times these of $(z, \theta) \in \mathbb{R}^{2} \mapsto B P v(z, \theta)$. As these latter coefficients are $v_{n, k} e^{-\sqrt{n^{2}+k^{2}}}$, we know that

$$
\left(v_{n, k} e^{(1+2 \epsilon)|n|-\sqrt{n^{2}+k^{2}}}\right)_{n, k} \in l^{2}\left(\mathbb{Z}^{2}\right) .
$$

Now for small enough $\eta$, if $|k| \leq \eta|n|, \sqrt{n^{2}+k^{2}} \leq(1+\epsilon)|n|$. Therefore,

$$
\left(v_{n, k} e^{\epsilon|n|}\right)_{n, k} \in l^{2}\left(\left\{(n, k) \in \mathbb{Z}^{2}|| k|\leq \eta| n \mid\right\}\right) .
$$

\subsection{Proof of Lemma 3.2}

The proof of this lemma uses the same ingredients as the proof of Proposition 2.2. We will use the same kind of biorthogonal sequence. Though, in that case, we will use it for the high frequencies, for which the geometrical control condition holds. This is why the norms of the functions $h$ are not growing exponentially. Another difference lies in the fact that for high frequencies, we have to sum an infinite number of values of $k$ for each $n$. $(k \geq \eta|n|)$. So we have to take care of the convergence of the series, which compels us to be careful about the constants.

We will prove that for any positive $\eta$, we can find a positive time $T(\eta)$ and a sequence of functions $h_{\eta}^{n, k}$, chosen even or odd, such that

(i) for any $(n, k)$ in $\mathbb{Z}^{2}, h_{\eta}^{n, k}$ is supported by $[-T(\eta), T(\eta)]$,

(ii) for any $(n, k)$ in $\mathbb{Z}^{2},\left\|h_{\eta}^{n, k}\right\|_{L^{2}} \leq \frac{C}{(1+|n|+|k|)^{N}}$,

(iii) if $k \neq k_{0}, \int h_{\eta}^{n, k_{0}}(t) e^{ \pm i \sqrt{n^{2}+k^{2}}}=0$,

(iv) if $|n| \leq \eta|k|,\left|\int h_{\eta}^{n, k_{0}}(t) e^{ \pm i \sqrt{n^{2}+k^{2}}}\right| \geq \frac{C}{(1+|n|+|k|)^{N}}$.

Let us see how this allows us to prove Lemma 3.2. 
Let $\underline{u}$ be an element of $E_{0}$, and put $\underline{u}=\sum_{m, k}\left(\alpha_{m, k}^{1} e_{m, k}^{1}+\alpha_{m, k}^{2} e_{m, k}^{2}\right)$.

For any $\left(n, k_{0}\right)$ such that $\left|k_{0}\right| \geq \eta|n|$ and any integer $M$ bigger than $n$,

$$
\begin{aligned}
\int h_{e_{\eta}}^{k_{0}, n}(t) e^{-i n \theta} K & {\left[\sum_{m, j=1}^{M}\left(\alpha_{m, j}^{1} e_{m, j}^{1}+\alpha_{m, j}^{2} e_{m, j}^{2}\right)\right](t, \theta) d t d \theta } \\
= & \int h_{e_{\eta}}^{k_{0}, n}(t) e^{-i n \theta} \sum_{j=1}^{M}\left[\alpha_{n, j}^{1} K e_{n, j}^{1}(t, \theta)+\alpha_{n, j}^{2} K e_{n, j}^{2}(t, \theta)\right] d t d \theta \\
= & \sum_{j=1}^{M}\left[\alpha_{n, j}^{1} \int h_{e}^{k_{0}, n}(t) e^{-i n \theta} K e_{n, j}^{1}(t, \theta) d t d \theta+\alpha_{n, j}^{2} \int h_{e}{ }_{e}^{k_{0}, n}(t) e^{-i n \theta} K e_{n, j}^{2}(t, \theta) d t d \theta\right] \\
= & \frac{1}{\sqrt{2 \pi}} \sum_{j=1}^{M}\left[\alpha_{n, j}^{1} \frac{j}{\sqrt{1+j^{2}+n^{2}}} \int h_{e_{\eta}}^{k_{0}, n}(t)\left(e^{i t \sqrt{j^{2}+n^{2}}}+e^{-i t \sqrt{j^{2}+n^{2}}}\right) d t\right. \\
& \left.+\alpha_{n, j}^{2} \frac{j}{i \sqrt{n^{2}+j^{2}}} \int h_{e_{\eta}}^{k_{0}, n}(t)\left(e^{i t \sqrt{j^{2}+n^{2}}}-e^{-i t \sqrt{j^{2}+n^{2}}}\right) d t\right] .
\end{aligned}
$$

So, as ${\widehat{h_{e \epsilon, q}}}_{e_{0}, n}$ is even and ${\widehat{h_{o \epsilon, q}}}_{k_{0}, n}^{k^{\prime}}$ is odd,

$$
\begin{aligned}
\int h_{e \eta}^{k_{0}, n}(t) e^{-i n \theta} K\left[\sum_{m, j=1}^{M}\left(\alpha_{m, j}^{1} e_{m, j}^{1}+\alpha_{m, j}^{2} e_{m, j}^{2}\right)\right] & (t, \theta) d t d \theta \\
& =\sqrt{\frac{2}{\pi}} e^{i n \theta} \sum_{j=1}^{M} \alpha_{n, j}^{1} \frac{j}{\sqrt{1+j^{2}+n^{2}}} \int h_{e}^{k_{0}, n}(t) e^{i t \sqrt{j^{2}+n^{2}}} d t .
\end{aligned}
$$

So by (iii), if $M \geq k_{0}$,

$$
\begin{aligned}
\int h_{e \eta}^{k_{0}, n}(t) e^{-i n \theta} K\left[\sum_{m, j=1}^{M}\left(\alpha_{m, j}^{1} e_{m, j}^{1}+\alpha_{m, j}^{2} e_{m, j}^{2}\right)\right] & (t, \theta) d t d \theta \\
& =\sqrt{\frac{2}{\pi}} \alpha_{n, k_{0}}^{1} \frac{k_{0}}{1+k_{0}^{2}+n^{2}} \int h_{e_{\eta}}^{k_{0}, n}(t) e^{i t \sqrt{k_{0}^{2}+n^{2}}} d t .
\end{aligned}
$$

So by (iv), as $\left|k_{0}\right| \geq \eta|n|$,

$$
\left|\int h_{e_{\eta}, n}^{k_{0}, n}(t) e^{-i n \theta} K\left[\sum_{m, j=1}^{M}\left(\alpha_{m, j}^{1} e_{m, j}^{1}+\alpha_{m, j}^{2} e_{m, j}^{2}\right)\right](t, \theta) d t d \theta\right| \geq\left|\alpha_{n, k_{0}}^{1}\right| \frac{c}{(1+|n|+|k|)^{N}} .
$$

Therefore, if $M$ goes to the infinity,

$$
\left|\int h_{e \eta}^{k_{0}, n}(t) e^{-i n \theta} K \underline{u}(t, \theta) d t d \theta\right| \geq\left|\alpha_{n, k_{0}}^{1}\right| \frac{c}{(1+|n|+|k|)^{N}} .
$$


Similarly

$$
\left|\int h_{o_{\eta}, n}^{k_{0}, n}(t) e^{-i n \theta} K \underline{u}(t, \theta) d t d \theta\right| \geq\left|\alpha_{n, k_{0}}^{2}\right| \cdot \frac{c}{(1+|n|+|k|)^{N}} .
$$

Now by (i),

$$
\left|\int h_{\eta}^{k_{0}, n}(t) e^{-i n \theta} K \underline{u}(t, \theta) d t d \theta\right|^{2} \leq \int\left|h_{\eta}^{k_{0}, n}(t)\right|^{2} d t \int_{\mathbb{S}^{1} \times(-T(\eta), T(\eta))}|K \underline{u}(t, \theta)|^{2} d t d \theta .
$$

So by (ii),

$$
\left|\int h_{\eta}^{k_{0}, n}(t) e^{-i n \theta} K \underline{u}(t, \theta) d t d \theta\right|^{2} \leq C(1+|n|+|k|)^{N} \int_{\mathbb{S}^{1} \times(-T(\eta) T(\eta))}|K \underline{u}(t, \theta)|^{2} d t d \theta .
$$

Thus by (3.1) and (3.2),

$$
\left|\alpha_{n, k_{0}}^{i}\right|^{2} \leq C(1+|n|+|k|)^{2 N}|K \underline{u}(t, \theta)|_{L^{2}(\Gamma \times(-T(\eta), T(\eta)))}^{2} .
$$

\subsection{Construction of functions $h_{\eta}^{n, k}$}

We will build the sequence $h_{\eta}^{n, k}$ with the same ingredients as the sequence $h_{\epsilon, q}^{n, k}$. Recall that we defined functions $g$ by

$$
\begin{aligned}
g_{+T, q}^{n}(t) & =\mathbf{1}_{(-T, T)} e^{i n \frac{T}{\delta x_{q}} h_{q}\left(\frac{x_{q}}{T} t\right)}, \\
g_{e T, q}^{n}(t) & =\mathbf{1}_{(-T, T)} \cos \left(n \frac{T}{\delta x_{q}} h_{q}\left(\frac{x_{q}}{T} t\right)\right), \\
g_{o T, q}^{n}(t) & =\mathbf{1}_{(-T, T)} \sin \left(n \frac{T}{\delta x_{q}} h_{q}\left(\frac{x_{q}}{T} t\right)\right) .
\end{aligned}
$$

But this time, we will put $\sqrt{1+\eta^{2}}>\frac{1}{\delta}>1$. The following lemma holds

Lemma 3.4. For big enough $T$, there are three positive real constants $C_{q}^{1}, C_{q, T}^{2}, c_{q, T, \delta}^{3}$ and an integer $N$ such that

- for any integer $n$ and any real number $\tau$ in $\left[-\frac{n}{\delta}, \frac{n}{\delta}\right]$,

$$
\left|\widehat{g}_{p, q}^{n}(\tau)\right| \leq C_{q, T}^{2} e^{-T n C_{q}^{1}\left(\frac{1}{\delta}-\left|\frac{\tau}{n}\right|\right)^{\frac{q}{q-1}}} .
$$

- For any integers $n$ and $k_{0}$ such that $\left|k_{0}\right| \geq \eta|n|$, there is a time $T_{n, k_{0}}$ in $[T, T+1]$ such that

$$
\left.\mid \widehat{g}_{p_{T_{n, k_{0}}, q}}^{n}\left( \pm \sqrt{n^{2}+k_{0}^{2}}\right)\right) \mid \geq \frac{c_{q, T, \delta}^{3}}{\left(1+|n|+\left|k_{0}\right|\right)^{N}} .
$$

We shall now fix $q=3$. We take $T(\eta)$ large enough to ensure $C_{q}^{1}\left(\frac{1}{\delta}-1\right) \geq 3 \pi$. Then, we define the times $T_{\eta}^{n, k}$ by taking the time given by Lemma 3.4 if $|k| \geq \eta|n|$ and putting $T_{\eta}^{n, k}=T(\eta)$ otherwise. Then we define

$$
\begin{aligned}
h_{p_{\eta}}^{n, k} & =\widehat{f}^{k, n} \cdot \widehat{g}_{p_{\eta}^{n, k}, q}^{n}(\tau), \\
h_{i \eta}^{n, k} & =\widehat{f}^{k, n} \cdot \widehat{g}_{i_{T_{\eta}, q}^{n, k}}^{n}(\tau) .
\end{aligned}
$$


Let us check properties (i) to (iv)

(i) By convolution, $h$ is supported by $\left[-T^{\prime}(\eta), T^{\prime}(\eta)\right]$ with $T^{\prime}(\eta)=T(\eta)+1+3 \pi$.

(ii) Once again, the problem is concentrated in $[-n, n]$, the rest being easily bounded by polynomials. Now over $\frac{\tau}{n} \in[-1,1]$, by $(2.4)$,

So by Lemma 3.4 , as $\frac{1}{\delta}>1$, for $\left|\frac{\tau}{n}\right| \leq 1$,

$$
\left|\widehat{f}^{k_{0}, n}(\tau)\right|^{2} \leq C \quad e^{2 \pi n \sqrt{1-\left|\frac{\tau}{n}\right|^{2}}} \leq C e^{2 \pi n} .
$$

$$
\int_{-n}^{n}\left|\widehat{h}_{\eta}^{k, n}\right|^{2} d \tau \leq C
$$

Hence

$$
\left\|\widehat{h}_{\eta}^{k_{0}, n}\right\|_{L^{2}}^{2} \leq C(1+|n|+|k|)^{N}
$$

(iii) is a direct consequence of the properties of $f$

(iv) is a direct consequence of (f-iv) and Lemma 3.4.

We only have the proof of Lemma 3.4 left.

\subsection{Proof of Lemma 3.4}

Once again, we have to estimate an integral with either a stationary or a non-stationary phase. The first estimate in non stationary, and it has been already proved in the case of Lemma 2.6. The second estimate is a stationary phase formula. Remind that we consider

$$
\phi(\alpha, \beta)=\int \theta_{q}^{\prime}(v) e^{i \alpha\left[v-\beta \theta_{q}(v)\right]} d v
$$

with $\alpha \rightarrow+\infty$.

The stationary phase formula shows that

$$
\phi(\alpha, \beta)=\cos \alpha p_{0}(\beta, \delta)\left[\frac{\theta_{q}^{\prime}\left(v_{0}(\beta, \delta)\right)}{\sqrt{H_{\beta, \delta}} \sqrt{\alpha}}+\sum_{j=1}^{N} c_{j} \frac{\theta_{q}^{(2 j+1)}\left(v_{0}(\beta, \delta)\right)}{\alpha^{j} H_{\beta, \delta}^{j} \sqrt{\alpha H_{\beta, \delta}}}\right]+r_{\beta, \delta}(\alpha)
$$

where $r_{\beta, \delta}(\alpha) \leq \frac{C_{\beta, \delta}}{\left(H_{\beta, \delta} \alpha\right)^{N+1}}$. $H_{\beta, \delta}$ denotes the Hessian at the critical points.

The value $C_{\beta, \delta}$ depends only on the first $2 N+1$ derivatives of $v \mapsto \theta_{q}(v)$ at $v=v_{0}(\beta, \delta)$.

We know that

$$
v_{0}(\beta, \delta)=\frac{1}{\delta x_{q}}(\delta \beta-1)^{\frac{1}{q-1}} .
$$

Now if $\beta$ takes the values $\frac{\sqrt{n^{2}+k^{2}}}{n}$ with $|k| \geq \eta|n|,(\delta \beta-1)$ remains in a set $[\epsilon,+\infty)$ with $\epsilon>0$, therefore, $H_{\beta, \delta}$ remains in an interval of the same type. Furthermore, all derivatives of $\theta_{q}$ are bounded. So for big enough $\alpha$ and any value of $(n, k)$ such that $|k| \geq \eta|n|$,

$$
\begin{gathered}
\left|\frac{\theta_{q}^{\prime}\left(v_{0}(\beta, \delta)\right)}{\sqrt{\alpha}}+\sum_{j=1}^{N} \frac{\theta_{q}^{(2 j+1)}\left(v_{0}(\beta, \delta)\right)}{\alpha^{j} \sqrt{\alpha}}\right| \geq \frac{\left|\theta_{q}^{\prime}\left(v_{0}(\beta, \delta)\right)\right|}{2 \sqrt{\alpha}}, \\
\left|r_{\beta, \delta}(\alpha)\right| \leq c_{\delta}^{\prime} \frac{\theta_{q}^{\prime}\left(v_{0}(\beta, \delta)\right)}{4 \sqrt{\alpha} \sqrt{H_{\beta, \delta}}}
\end{gathered}
$$


And for the same reason, we have $\left|p_{0}(\beta, \delta)\right| \geq c_{\delta}>0$, and therefore for any given $T>0$, we can pick a time $T_{n, k_{0}}$ in $[T, T+1]$ such that

$$
\cos \left(n T_{n, k_{0}} p_{0}\left(\frac{\sqrt{n^{2}+k_{0}^{2}}}{n}, \delta\right)\right) \geq c_{\delta}^{\prime} .
$$

Therefore we have

$$
\left|\phi\left(n T_{n, k_{0}}, \frac{\sqrt{n^{2}+k_{0}^{2}}}{n}\right)\right| \geq \frac{c_{\delta}^{\prime} \theta_{q}^{\prime}\left(v_{0}\left(\frac{\sqrt{n^{2}+k_{0}^{2}}}{n}, \delta\right)\right)}{4 \sqrt{H} \sqrt{n} \sqrt{T_{n, k_{0}}}} \geq \frac{c}{(1+|n|+|k|)^{N}} .
$$

Which means that

$$
\left|{\widehat{g_{+}}}_{n, k_{0}, q}^{n}\left(\sqrt{n^{2}+k_{0}^{2}}\right)\right| \geq \frac{C}{(1+|n|+|k|)^{N}} .
$$

The exact value of $N$ depends on the power of $\beta$ that appears in the Hessian. It could be computed, which would lead to a control result for Sobolev functions instead of $C^{\infty}$ ones. Though, as the result would certainly not be optimal in term of Sobolev power, it would be a little bit artificial.

\section{Proof of Theorem 1.2}

In order to prove this theorem, we will characterize the spaces $G_{\alpha}$ and $F_{T}$ by their images by the operator $B$. Roughly speaking, these images will be spaces of functions that are holomorphic over given sets, that we will compute. By comparing these sets will be able to compare the initial spaces.

Before we can use the operator $B$, we have to continue the functions we consider over $\mathbb{R}^{2}$. In order to to this, we will periodise the system.

We are currently considering the solutions $u_{1}(z, \theta, t)$ of

$$
\mid \begin{aligned}
& \square u_{1}=0 \text { over }(0, \pi) \times \mathbb{S}^{1} \times \mathbb{R}, \\
& \left.u_{1}\right|_{z=0}=g(\theta, t) \in L^{2} \text { supported by }\left.t \in[0, T] u_{1}\right|_{z=\pi}=0, \\
& \left.\left(u_{1}, \partial_{t} u_{1}\right)\right|_{t=T}=0 .
\end{aligned}
$$

We look for the space $F_{T}$ spanned by $\left.\left(u_{1}, \partial_{t} u_{1}\right)\right|_{t=0}$.

This problem can be continued antisymetrically to values of $z$ in $[0,2 \pi]$ by putting $u_{2}(\pi+z, \theta, t)=-u_{2}(\pi-$ $z, \theta, t)$.

We get the following problem

$$
\mid \begin{aligned}
& \square u_{2}=0 \text { over }(0,2 \pi) \times \mathbb{S}^{1} \times \mathbb{R}, \\
& \left.u_{2}\right|_{z=0}=g(\theta, t) \text { supported by }[0, T], \\
& \left.u_{2}\right|_{z=2 \pi}=-g(\theta, t), \\
& \left.\left(u_{2}, \partial_{t} u_{2}\right)\right|_{t=T}=0 .
\end{aligned}
$$

To end with, we can take the $2 \pi$-periodic extension of $u_{2}$ with respect to $z$ in $\mathbb{R}$, that will be denoted $P u$.

$$
\begin{array}{r|l}
P u \text { is the solution of } & \begin{array}{l}
\square v=\left(g_{1} \delta_{z=0}+g_{2} \delta_{z=0}^{\prime}\right) *\left(\begin{array}{l}
\sum_{k \in \mathbb{Z}} \delta_{z=2 \pi k} \\
\left.\left(v, \partial_{t} v\right)\right|_{t>T}=0,
\end{array}\right.
\end{array},
\end{array}
$$

with $g_{1}=0$ and $g_{2}=2 g$ supported by $[0, T]$. 


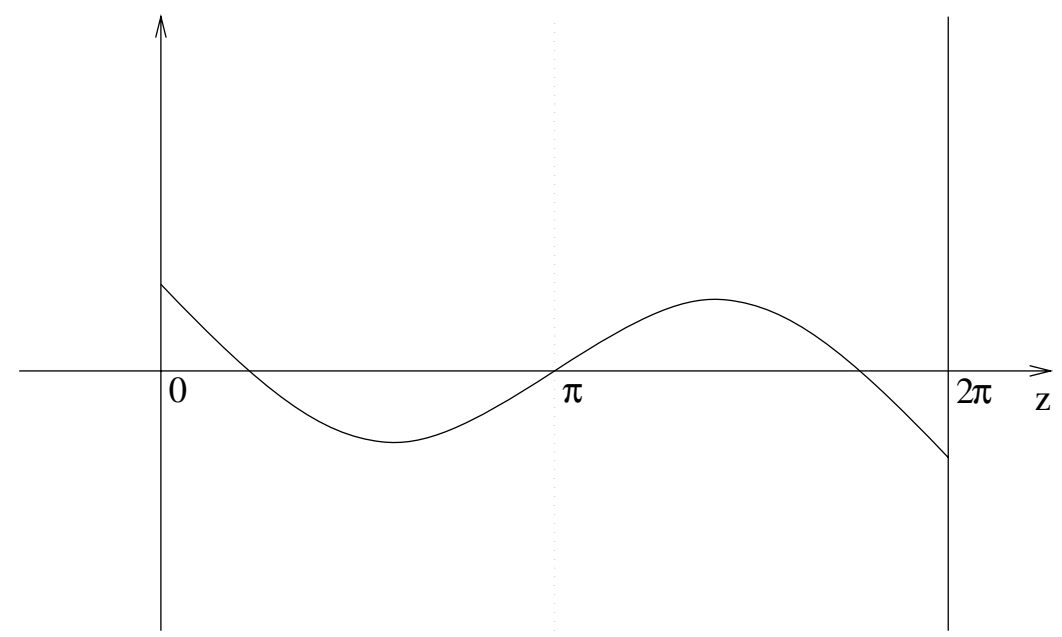

FIGURE 3. Antisymmetric continuation.

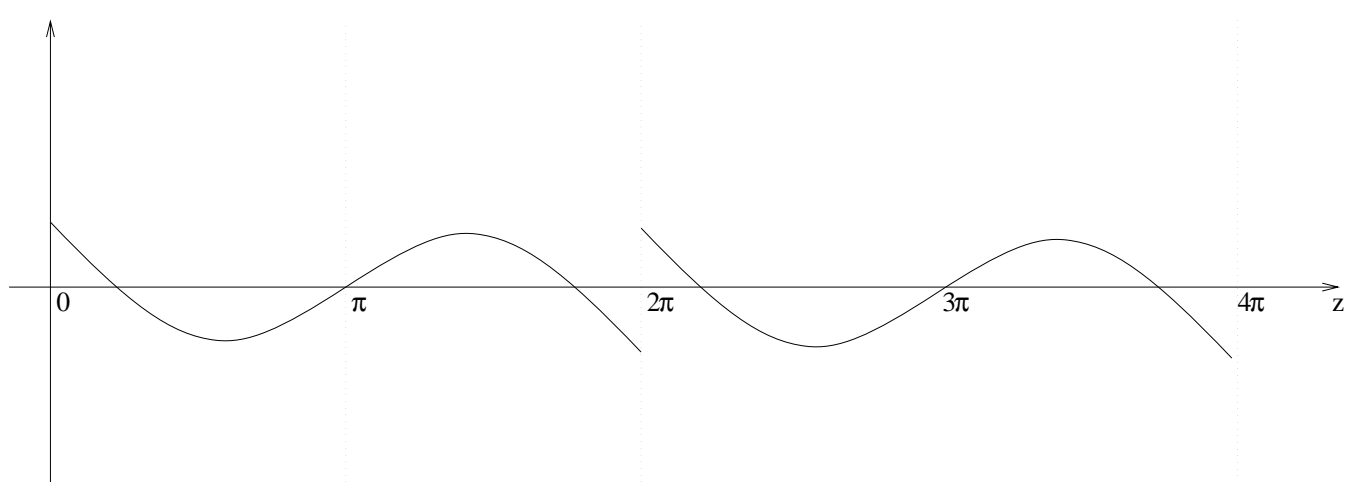

Figure 4. Periodic continuation.

Denote $\mathcal{F}_{T}$ the space spanned by $\left.\left(v, \partial_{t} v\right)\right|_{t=0}$ when $g_{1}, g_{2}$ are any distributions over $\mathbb{S}^{1} \times \mathbb{R}_{t}$ supported by $\{t \in[0, T]\}$.

Any element of $F_{T}$ is a couple of functions that we can periodise as shown before. Their periodised function belongs to $\mathcal{F}_{T}$. From now on, we will identify these functions with their image by periodisation. Then we can denote $F_{T} \subset \mathcal{F}_{T}$ instead of $P F_{T} \subset \mathcal{F}_{T}$. Notice that for any posive $\beta$ the Fourier series developpement of functions in $G_{\beta}$ is formally the same as the developpement of their periodised function, which makes the identification even easier.

In order to bound $\alpha_{C}(T)$ from bellow, we will compute the space $\mathcal{F}_{T}$, thanks to an explicit Fourier integral operator. Then we will find a $G_{\beta}$ that won't be a subset of $\mathcal{F}_{T}$, hence not a subset of $F_{T}$ either.

\subsection{Two lemmas}

Recall that

$$
B f(z)=\int e^{i z \cdot \xi} \widehat{f}(\xi) e^{-|\xi|} d \xi .
$$


Let us denote $\mathcal{E}_{a} \subset \mathbb{C}^{2}$ the following domain, which is independent from the real parts of the variables

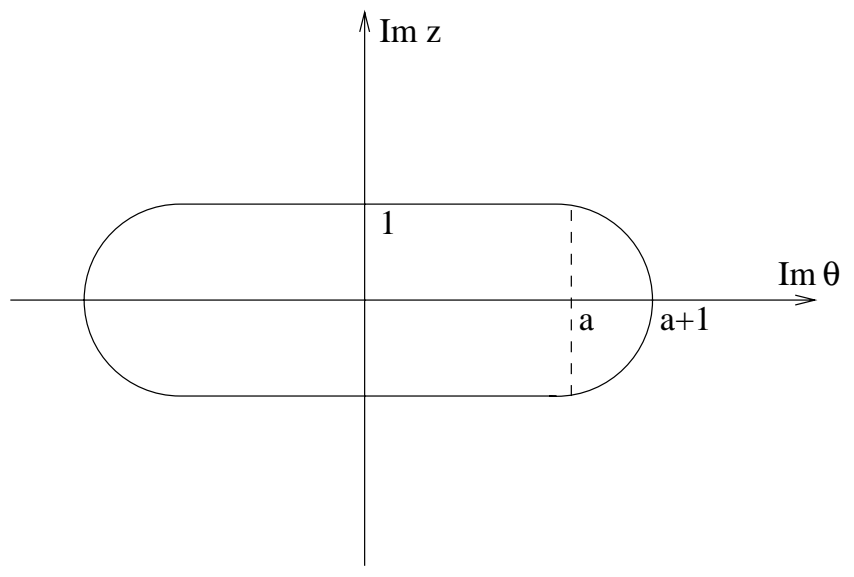

Figure 5. The set $\mathcal{E}_{a}$.

We will also denote $\mathbb{R}^{2} \ni(z, \theta) \in \mathcal{R} E_{a}$ if $(i z, i \theta) \in \mathcal{E}_{a}$.

Here are the main two lemmas of the proof.

Lemma 4.1. For any real number $\alpha$ and any $\left(z_{0}, \theta_{0}\right)$ in $\mathbb{R}^{2}$, there is a function $v$ in $G_{0, \alpha}$ such that

$$
B v \in \mathcal{O}\left(\mathcal{E}_{\alpha}\right) \text { and }
$$

Bv has no holomorphic continuation at $\left(z_{0}, \theta_{0}+i(\alpha+1)\right)$.

Lemma 4.2. For any real time $T$, there is a connected open domain $\Omega_{T}$ of $\mathbb{C}^{2}$ that contains $\mathcal{E}_{0}$ over which any function of $\mathrm{B \mathcal {F }}_{T}$ has an holomorphic continuation.

It contains all the points $\left(\pi, \theta_{1}-i \epsilon\right)$ for $\epsilon \in(0,1)$ and

$$
\theta_{1}=i \frac{\sqrt{2}}{2}\left[1-T^{2}+\pi^{2}+\sqrt{\left(T^{2}-1-\pi^{2}\right)^{2}+4 T^{2}}\right]^{\frac{1}{2}}
$$

Let us see how we can conclude with those to lemmas. Let $\epsilon$ be a positive number smaller than 1. Put $\beta=\Im m \theta_{1}-1-\epsilon$.

Let $v$ be the function of $G_{0, \beta}$ given by Lemma 4.1 for $z_{0}=\pi$ and $\theta_{0}=0$.

It has no holomorphic continuation in a neighborhood of $\left(\pi, \theta_{1}-i \epsilon\right)$.

Now Lemma 4.2 proves that any function of $B \mathcal{F}_{T}$ has an holomorphic continuation at that point.

So for instance $(v, 0)$ does not belong to $\mathcal{F}_{T}$. Therefore $G_{\beta} \not \subset \mathcal{F}_{T}$.

By definition of $\alpha_{C}(T)$, this proves that

$$
\alpha_{C}(T) \geq \frac{\sqrt{2}}{2}\left[\sqrt{T^{4}+2\left(1-\pi^{2}\right) T^{2}+\left(1+\pi^{2}\right)^{2}}-T^{2}+\pi^{2}+1\right]^{\frac{1}{2}}-1 \approx \frac{\pi^{2}}{2 T^{2}}
$$




\subsection{Proof of Lemma 4.1}

Let us begin with a technical remark.

For any $\left(z_{0}, \theta_{0}\right)$ in $\mathbb{R}^{2}$, put $\phi_{\alpha}(n, k)=n \theta_{0}+k z_{0}-\sqrt{n^{2}+k^{2}}-\alpha|n|$.

Remark 4.3. For any positive $\alpha$, any $\beta$ smaller than 1 , any positive $\alpha^{\prime}$ smaller than $\alpha$ and any $\left(\theta_{0}, z_{0}\right)$ in $\mathcal{R} E_{\alpha^{\prime}}$ such that $\left|z_{0}\right| \leq \beta$, there is a positive constant $C_{\alpha-\alpha^{\prime}, \beta}$ such that for any $(n, k)$ in $\mathbb{R}^{2}$,

$$
\phi_{\alpha}(n, k) \leq-C_{\alpha-\alpha^{\prime}, \beta} \sqrt{n^{2}+k^{2}} .
$$

Proof. Take for instance $\theta_{0} \geq 0$ and $n \geq 0$.

$$
\begin{aligned}
\phi_{\alpha}(n, k) & =n \theta_{0}+k z_{0}-\sqrt{n^{2}+k^{2}}-\alpha|n| \\
& =\sqrt{n^{2}+k^{2}}\left[\frac{n}{\sqrt{n^{2}+k^{2}}}\left(\theta_{0}-\alpha\right)+\frac{k}{\sqrt{n^{2}+k^{2}}} z_{0}-1\right] \\
& =\left|\begin{array}{c}
n \\
k
\end{array}\right|\left[\vec{e}_{n, k} \cdot\left(\begin{array}{c}
\theta_{0}-\alpha \\
z_{0}
\end{array}\right)-1\right] .
\end{aligned}
$$

Now $\left(z_{0}, \theta_{0}\right) \in \mathcal{R} E_{\alpha^{\prime}}$, and $\left|z_{0}\right|<1$.

If we put

$$
C_{\alpha-\alpha^{\prime}, \beta}^{\prime}=\sup \left\{(\theta-\alpha, z) \cdot \vec{e}_{\eta} ;(\theta, z) \in \mathcal{R} E_{\alpha^{\prime}},|z|<\beta, \vec{e}_{\eta} \in \mathbb{S} \cap\{n>0\}\right\},
$$

we get $C_{\alpha-\alpha^{\prime}, \beta}^{\prime}<1$.

Then $\phi_{\alpha}(n, k) \leq-C_{\alpha-\alpha^{\prime}, \beta} \sqrt{n^{2}+k^{2}}$, where $C_{\alpha-\alpha^{\prime}, \beta}=1-C_{\alpha-\alpha^{\prime}, \beta}^{\prime}$.

Lemma 4.4. For any positive $\alpha$ and $j=0,1$,

$$
B\left(G_{j, \alpha}\right) \subset \mathcal{O}\left(\mathcal{E}_{\alpha}\right)
$$

Proof. If $f(z, \theta)=\sum_{n, k} a_{n, k} e^{i n \theta+i k z-\alpha|n|} \in G_{j, \alpha}$,

$$
B f\left(z_{r}+i z_{i}, \theta_{r}+i \theta_{i}\right)=\sum_{n, k} a_{n, k} e^{i n \theta_{r}+i k z_{r}-n \theta_{i}-k z_{i}-\sqrt{n^{2}+k^{2}}-\alpha|n|} .
$$

Then Remark 4.3 indicates that $B f$ is defined by an exponentially decreasing series which sum is an holomorphic function, that is the only possible holomorphic continuation to $B f$.

We have shown that $B G_{\alpha}$ is a subset of $\mathcal{O}\left(\mathcal{E}_{\alpha}\right)^{2}$.

To show Lemma 4.1, we still have to build a function that isn't holomorphic at the right place. This is done in the following lemma

Lemma 4.5. For any positive $\alpha$ and any $\left(z_{0}, \theta_{0}\right)$ in $\mathbb{R}^{2}$, there is a function $v$ in $G_{0, \alpha}$ such that

$$
\text { Bv has no holomorphic continuation at }\left(z_{0}, \theta_{0}+i(\alpha+1)\right) \text {. }
$$

Proof. Let $v$ be an element of $\mathcal{S}^{\prime}\left(\mathbb{R}^{2}\right)$, defined by

$$
v=\sum_{n, k \leq 0} \frac{1}{\langle n\rangle} \frac{1}{\langle k\rangle} e^{-|n| \alpha+i n\left(\theta-\theta_{0}\right)+i k\left(z-z_{0}\right)}
$$


where $\langle k\rangle=\sup (1,|k|)$.

$$
v \in G_{0, \alpha} .
$$

We know by Lemma 4.4 that $B v$ has an unique holomorphic continuation over $\mathcal{E}_{\alpha}$. Let us compute it.

$$
B v\left(\theta_{r}+i \theta_{i}, z_{r}+i z_{i}\right)=\sum_{n, k \leq 0} \frac{1}{\langle n\rangle\langle k\rangle} e^{-\alpha|n|+i\left(\theta_{r}-\theta_{0}\right) n+i\left(z_{r}-z_{0}\right) k-\theta_{i} n-z_{i} k-\sqrt{n^{2}+k^{2}}} .
$$

So, as an holomorphic function over $\mathcal{E}_{\alpha}$,

$$
\frac{\partial^{3}}{\partial \theta^{3}} B v\left(\theta_{r}+i \theta_{i}, z_{r}+i z_{i}\right)=-i \sum_{n, k \leq 0} \frac{n^{3}}{\langle n\rangle\langle k\rangle} e^{-\alpha|n|+i\left(\theta_{r}-\theta_{0}\right) n+i\left(z_{r}-z_{0}\right) k-\theta_{i} n-z_{i} k-\sqrt{n^{2}+k^{2}}}
$$

Let us denote this this latter function $f$. We shall show that $f$ has no holomorphic continuation at $\left(\theta_{0}+i(\alpha)\right.$ $\left.+1), z_{0}\right)$.

In order to do this, we will find a sequence of points $\left(\theta^{n}, z^{n}\right)$ in $\mathcal{E}_{\alpha}$ that goes to $\left(\theta_{0}+i(\alpha+1), z_{0}\right)$ and such that $\left|f\left(\theta^{n}, z^{n}\right)\right| \rightarrow \infty$.

Hence $f$ cannot have any continuous continuation at $\left(\theta_{0}+i(\alpha+1), z_{0}\right)$ thus $B v$ has no holomorphic continuation there.

Let $\left(\theta_{0}^{\nu}, z_{0}^{\nu}\right)$ be the point $\left(\theta_{0}+i(\alpha+1-\nu), z_{0}\right)$.

For small enough $\nu,\left(\theta_{0}^{\nu}, z_{0}^{\nu}\right)$ belongs to $\mathcal{E}_{\alpha}$, so $f$ is defined at that point, and

$$
\text { if }\left(\theta_{0}^{\nu}, z_{0}^{\nu}\right)=\sum_{n, k \leq 0} \frac{n^{3}}{\langle n\rangle\langle k\rangle} e^{-\alpha|n|-\theta_{i}^{\nu} n-z_{i}^{\nu} k-\sqrt{n^{2}+k^{2}}} .
$$

All terms in that sum are positive, so

$$
\text { if }\left(\theta_{0}^{\nu}, z_{0}^{\nu}\right) \geq \sum_{n \leq-1} n^{2} e^{n \alpha-(\alpha+1-\nu) n-|n|}=\sum_{n=1}^{+\infty} n^{2} e^{-\nu n} .
$$

Thus

$$
\lim _{\nu \rightarrow 0} i f\left(\theta_{0}^{\nu}, z_{0}^{\nu}\right)=+\infty
$$

This proves the lemma.

\subsection{Proof of Lemma 4.2}

Time being reversible in the wave equation, $\mathcal{F}_{T}$ can also be defined that way.

Let $g_{1}, g_{2}$ be in $\mathcal{D}^{\prime}\left(\mathbb{S}^{1} \times \mathbb{R}_{t}\right)$ supported by $\{t \in[0, T]\}$ and let $v$ be the solution of $\square v=\underbrace{\left(g_{1} \delta_{z=0}+g_{2} \delta_{z=0}^{\prime}\right) * \sum_{k \in \mathbb{Z}} \delta_{z=2 \pi k}}_{g(z, t)}$ with $\left.\left(v, \partial_{t} v\right)\right|_{t=0}=0$.

Put $S(g)=\left.\left(v, \frac{\partial v}{\partial t}\right)\right|_{t=T}$.

$$
\mathcal{F}_{T}=\operatorname{Im} S \text {. }
$$


Now we know a solution to the elementary equation $\square e=\delta$ with $\left.e\right|_{t<0}=0$

$$
e=\frac{1}{4 \pi^{2}} \int e^{i x \cdot \xi} \mathbf{1}_{t>0} \frac{\sin t|\xi|}{|\xi|} d \xi
$$

So by convolution,

$$
S(g)(\theta, z)=\left(\begin{array}{l}
\iint \frac{\sin (T-s)|\xi|}{|\xi|} e^{i\left(\theta-\theta^{\prime}\right) \xi_{1}+i\left(z-z^{\prime}\right) \xi_{2}} g\left(z^{\prime}, \theta^{\prime}, s\right) d z^{\prime} d \theta^{\prime} d s d \xi \\
\iint \cos (T-s)|\xi| e^{i\left(\theta-\theta^{\prime}\right) \xi_{1}+i\left(z-z^{\prime}\right) \xi_{2}} g\left(z^{\prime}, \theta^{\prime}, s\right) d z^{\prime} d \theta^{\prime} d s d \xi
\end{array}\right)
$$

Then

$$
B S(g)(\theta, z)=\left(\begin{array}{c}
\int_{0}^{T} \int \frac{\sin (T-s)|\xi|}{|\xi|} e^{i\left(\theta-\theta^{\prime}\right) \xi_{1}+i\left(z-z^{\prime}\right) \xi_{2}-|\xi|} g\left(z^{\prime}, \theta^{\prime}, s\right) d z^{\prime} d \theta^{\prime} d s d \xi \\
\int_{0}^{T} \int \cos (T-s)|\xi| e^{i\left(\theta-\theta^{\prime}\right) \xi_{1}+i\left(z-z^{\prime}\right) \xi_{2}-|\xi|} g\left(z^{\prime}, \theta^{\prime}, s\right) d z^{\prime} d \theta^{\prime} d s d \xi
\end{array}\right)
$$

As $\sin x=\frac{1}{2 i}\left(e^{i x}-e^{-i x}\right)$, and $\cos x=\frac{1}{2}\left(e^{i x}+e^{-i x}\right)$, with a change of variables, we can rewrite

$$
B S(g)(\theta, z)=\left(\begin{array}{l}
\int_{0}^{2 T} \int \frac{e^{i(T-s)|\xi|}}{2 i|\xi|} e^{i\left(\theta-\theta^{\prime}\right) \xi_{1}+i\left(z-z^{\prime}\right) \xi_{2}-|\xi|} h_{1}\left(z^{\prime}, \theta^{\prime}, s\right) d z^{\prime} d \theta^{\prime} d s d \xi \\
\int_{0}^{2 T} \int \frac{e^{i(T-s)|\xi|}}{2} e^{i\left(\theta-\theta^{\prime}\right) \xi_{1}+i\left(z-z^{\prime}\right) \xi_{2}-|\xi|} h_{2}\left(z^{\prime}, \theta^{\prime}, s\right) d z^{\prime} d \theta^{\prime} d s d \xi
\end{array}\right)
$$

where

$$
h_{1}\left(z^{\prime}, \theta^{\prime}, s\right)=h_{2}\left(z^{\prime}, \theta^{\prime}, s\right)=g\left(z^{\prime}, \theta^{\prime}, s\right) \text { if } 0 \leq s \leq T
$$

and

$$
h_{1}\left(z^{\prime}, \theta^{\prime}, s\right)=-h_{2}\left(z^{\prime}, \theta^{\prime}, s\right)=g\left(z^{\prime}, \theta^{\prime}, 2 T-s\right) \text { if } T \leq s \leq 2 T .
$$

$B S(g)$ is the image of $g$ by a Fourier Integral Operator with complex phase. As this operator is explicit, it is easy to compute the set over which $B S(g)$ is always holomorphic. We can do this by computing at first the domain of holomorphy for the kernel, and then compute an envelope.

Lemma 4.6. Holomorphy of the kernel.

The function $z \mapsto \int e^{i z \cdot \xi+(i t-1)|\xi|} d \xi$, that will be denoted $f_{t}(z)$ can be holomorphicaly continued in the neighborhood of any point of the domain $\mathbb{C}^{2} \backslash\left\{z \in \mathbb{C}^{2} \mid z^{2}=(t+i)^{2}\right\}$ as the function

$$
f_{t}(z)=\frac{2 i \pi(t+i)}{\left[(t+i)^{2}-z^{2}\right]^{\frac{3}{2}}} .
$$


Proof. For $|\Im m z|<1$,

$$
\begin{aligned}
f_{t}(z) & =\iint e^{i\left(z_{1} \xi_{1}+z_{2} \xi_{2}\right)+(i t-1) \sqrt{\xi_{1}^{2}+\xi_{2}^{2}}} d \xi_{1} d \xi_{2} \\
& =\int_{0}^{2 \pi} \int_{0}^{+\infty} e^{i \rho \cos \theta z_{1}+i \rho \sin \theta z_{2}+(i t-1) \rho} \rho d \rho d \theta \\
& =\int_{0}^{2 \pi} \frac{-d \theta}{\left(\cos \theta z_{1}+\sin \theta z_{2}+t+i\right)^{2}} \\
& =\int_{0}^{2 \pi} \frac{d \theta}{R(\theta)} .
\end{aligned}
$$

Remark. For $|\Im m z|<1, R(\theta)$ is never equal to zero because

Put $\eta=e^{i \theta}$.

$$
\left(\begin{array}{c}
\cos \theta \\
\sin \theta
\end{array}\right) \cdot\left(\begin{array}{c}
\Im m z_{1} \\
\Im m z_{2}
\end{array}\right)<1
$$

$d \eta=i e^{i \theta} d \theta, \cos \theta=\frac{1}{2}\left(\eta+\frac{1}{\eta}\right), \sin \theta=\frac{1}{2 i}\left(\eta-\frac{1}{\eta}\right)$.

$$
\begin{aligned}
f_{t}(z) & =\int_{\mathbf{U}} \frac{i d \eta}{\eta\left[\frac{z_{1} \eta+z_{1} / \eta}{2}+\frac{z_{2} \eta-z_{2} / \eta}{2 i}+t+i\right]^{2}} \\
& =\int_{\mathbf{U}} \frac{4 i \eta d \eta}{\left[\left(z_{1}-i z_{2}\right) \eta^{2}+2(t+i) \eta+z_{1}+i z_{2}\right]^{2}} \\
& =\int_{\mathbf{U}} \frac{4 i \eta d \eta}{P(\eta)}
\end{aligned}
$$

Remark. For $|\Im m z|<1, \eta \in \mathbb{U}, P(\eta)$ is never equal to zero because $R(\theta) \neq 0$.

The roots of $P(\eta)$ in $\mathbb{C}$ are

$$
\eta_{0}^{ \pm}=\frac{-(t+i) \pm \sqrt{(t+i)^{2}-z^{2}}}{z_{1}-i z_{2}} .
$$

These values are well defined if $z \in \mathbb{R}^{2} \backslash\{0\}$, and we can choose a determination for the square root when $|\Im m z|<1$ because $(t+i)^{2}-z^{2}$ is never zero.

Now let us restrict to the values of $z \in\left(\mathbb{R}^{2} \backslash\{0\}\right) \subset\{|\Im m z|<1\}$.

We shall show that $\left|\eta_{0}^{+}\right|<1$ and $\left|\eta_{0}^{-}\right|>1$.

We know that $\eta_{0}^{+} \eta_{0}^{-}=\frac{z_{1}+i z_{2}}{z_{1}-i z_{2}}$ which modulus is 1 because $z$ belongs to $\mathbb{R}^{2}$.

Moreover if $z_{2}^{0}=0$ and $\left|z_{1}^{0}\right|$ is small, $\left|\eta_{0}^{+}\left(z_{1}^{0}, z_{2}^{0}\right)\right|<1$ (so $\left|\eta_{0}^{-}\left(z_{1}^{0}, z_{2}^{0}\right)\right|>1$ ).

For $\left(z_{1}, z_{2}\right)$ in $\mathbb{R}^{2} \backslash\{0\}$, join $\left(z_{1}^{0}, z_{2}^{0}\right)$ to $\left(z_{1}, z_{2}\right)$ by a continuous path $\left.\left(z_{1}(\tau), z_{2}(\tau)\right)\right|_{\tau \in[0,1]}$ in $\mathbb{R}^{2} \backslash\{0\}$.

As $\left|\eta_{0}^{+}\left(z_{1}(\tau), z_{2}(\tau)\right)\right|$ is continuous, if $\left|\eta_{0}^{+}\left(z_{1}, z_{2}\right)\right| \geq 1$, there is a $\tau_{0} \in[0,1]$ such that

$$
\left|\eta_{0}^{+}\left(z_{1}\left(\tau_{0}\right), z_{2}\left(\tau_{0}\right)\right)\right|=1
$$

which is impossible because $P$ has no root over $\mathbb{U}$.

So for any $\left(z_{1}, z_{2}\right)$ in $\mathbb{R}^{2} \backslash\{0\},\left|\eta_{0}^{+}\left(z_{1}, z_{2}\right)\right|<1$ and $\left|\eta_{0}^{-}\left(z_{1}, z_{2}\right)\right|>1$.

So $f_{t}(z)$ is integrated over a contour that encloses only one pole. Thus

$$
f_{t}(z)=2 \pi \operatorname{Res}\left[\frac{4 i \eta}{P(\eta)} ; \eta_{0}^{+}\right]
$$




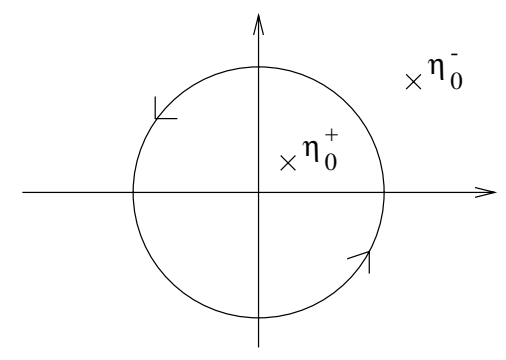

FIGURE 6. Integration contour.

So for any $z$ in $\mathbb{R}^{2} \backslash\{0\}$,

$$
f_{t}(z)=\frac{8 i \pi}{\left(z_{1}-i z_{2}\right)^{2}} \operatorname{Res}\left[\frac{\eta}{\left(\eta-\eta_{0}^{+}\right)^{2}\left(\eta-\eta_{0}^{-}\right)^{2}} ; \eta_{0}^{+}\right]=\frac{2 i \pi(t+i)}{\left[(t+i)^{2}-z^{2}\right]^{\frac{3}{2}}},
$$

where we have chosen a determination for the square root that remains fixed.

This function is holomorphic over the set $\{|\Im m z|<1\}$ and therefore is equal to $f_{t}$ there.

It can be continued as an holomorphic function to the set $\mathbb{C}^{2} \backslash\left\{z \in \mathbb{C}^{2} \mid z^{2}=(t+i)^{2}\right\}$ (or, more precisely over its simply connected bundle).

We shall now take the convolution product of this kernel. At first, let us consider only one Dirac function with respect to $z$ (e.g. ignore the periodicity with respect to $z$ ).

Consider the function

$$
\iint_{z^{\prime}, \theta^{\prime}} f_{T-s}\left(\theta-\theta^{\prime}, z-z^{\prime}\right) h_{i}\left(\theta^{\prime}, s\right) \delta_{z^{\prime}=0} d s d z^{\prime} d \theta^{\prime}
$$

with $h_{i}$ supported by $s \in[0,2 T]$. Let us compute at what point $(\theta, z)$ of $\mathbb{C}^{2}$ it can certainly be holomorphicaly continued.

Put $\theta=\theta_{r}+i \theta_{i} ; z=z_{r}+i z_{i}$.

According to Lemma 4.6, we must compute for any point $(\theta, z)$ if there is a $\left(\theta^{\prime}, s\right)$ in $\mathbb{R} \times[0,2 T]$ that ensures $(T-s+i)^{2}=\left(\theta-\theta^{\prime}\right)^{2}+z^{2}$.

The translation invariance with respect to $\theta_{r}$ is trivial, therefore we only have to compute if for given $\theta_{i}, z_{r}, z_{i}$ there is a $s$ in $[-T, T]$ and a $\theta_{r}$ in $\mathbb{R}$ such that

$$
(s+i)^{2}=\left(\theta_{r}+i \theta_{i}\right)^{2}+\left(z_{r}+i z_{i}\right)^{2} .
$$

Which means

$$
\left\{\begin{array}{c}
s^{2}-1=\theta_{r}^{2}-\theta_{i}^{2}+z_{r}^{2}-z_{i}^{2} \\
s=\theta_{i} \theta_{r}+z_{i} z_{r}
\end{array}\right.
$$

This will describe a geometric envelope inside which our function will be continued holomorphicaly as a sum of holomorphic functions. Of course, this set contains $\mathcal{E}_{0}$, so the envelope never gets inside this.

For given $\left(z_{r}, z_{i}\right)$ with $\left|z_{i}\right|<1$, let $\theta_{i}^{0}\left(z_{i}, z_{r}\right)$ be defined by

$$
\theta_{i}^{0}\left(z_{i}, z_{r}\right)=\inf \left\{\left|\theta_{i}\right| \operatorname{such} \text { that (4.4) has a solution with } s \in[-T, T], \theta_{r} \in \mathbb{R}\right\} .
$$

We have

$$
\theta_{i}^{0}\left(z_{i}, z_{r}\right) \geq \sqrt{1-\left|z_{i}\right|^{2}}>0
$$


- For $1>\left|z_{i}\right| \geq \frac{\left|z_{r}\right|}{T},(4.4)$ has a solution $\left(\theta_{r}, z_{r}\right)=s\left(\theta_{i}, z_{i}\right)$ where $s$ satisfies $z_{r}=s z_{i}$ and $\theta_{i}=\sqrt{1-\left|z_{i}\right|^{2}}$. Therefore

$$
1>\left|z_{i}\right| \geq \frac{\left|z_{r}\right|}{T} \Rightarrow \theta_{i}^{0}\left(z_{i}, z_{r}\right)=\sqrt{1-\left|z_{i}\right|^{2}}
$$

- For $\left|z_{i}\right|<\frac{\left|z_{r}\right|}{T}$, we can eliminate $\theta_{r}$ in (4.4), and get

$$
\theta_{i}^{4}+\left(s^{2}-1+z_{i}^{2}-z_{r}^{2}\right) \theta_{i}^{2}-\left(s-z_{r} z_{i}\right)^{2}=0 .
$$

As $\left|z_{i}\right|<1$, (4.7) has an unique positive root $\theta_{i}\left(z_{i}, z_{r}, s\right)$, that is analytic with respect to $s \in \mathbb{R}$, (4.5) shows that

$$
\theta_{i}^{0}\left(z_{i}, z_{r}\right)=\min _{s \in[-T, T]} \theta_{i}\left(z_{i}, z_{r}, s\right)
$$

Equations $\frac{\partial}{\partial s} \theta_{i}\left(z_{i}, z_{r}, s\right)=0$ and (4.7) prove that $s\left(1-\theta_{i}^{2}\right)=z_{r} z_{i}$ so $\left|z_{r} z_{i}\right| \leq|s|\left|z_{i}\right|^{2}$ according to (4.5), so $|s|>T$ for $\left|z_{i}\right| \neq 0$ because $\left|z_{i}\right|<\frac{\left|z_{r}\right|}{T}$.

If $z_{i}=0, \theta_{i}^{2}=1$ is not a solution to (4.7) (because $\left.\left|z_{r}\right|>0\right)$ and $\theta_{i}\left(0, z_{r}, 0\right)=\sqrt{1+z_{r}^{2}}>1=\lim _{s \rightarrow+\infty} \theta_{i}\left(0, z_{r}, s\right)$, so $s=0$ is a maximum.

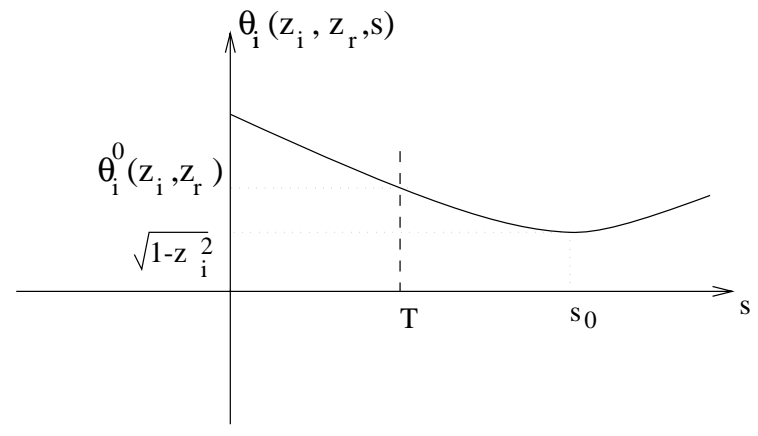

FiguRE 7

Thus

$$
\left|z_{i}\right|<\min \left(1, \frac{\left|z_{r}\right|}{T}\right) \Rightarrow \theta_{i}^{0}\left(z_{i}, z_{r}\right)=\frac{\sqrt{2}}{2}\left[1-T^{2}+z_{r}^{2}-z_{i}^{2}+\sqrt{\left(T^{2}-1+z_{i}^{2}-z_{r}^{2}\right)^{2}+4\left(T-\left|z_{r} z_{i}\right|\right)^{2}}\right]^{\frac{1}{2}} .
$$

If we put $\Omega_{T}\left(z_{r}\right)=\left\{\left(z_{i}, \theta_{i}\right)|| z_{i}|<1,| \theta_{i} \mid<\theta_{i}^{0}\left(z_{i}, z_{r}\right)\right\}$, the function

$$
\iint f_{T-s}\left(\theta-\theta^{\prime}, z-z^{\prime}\right) h_{i}\left(\theta^{\prime}, s\right) *\left(\sum_{k \in \mathbb{Z}} \delta_{z^{\prime}=2 \pi k}\right) d s d z^{\prime} d \theta^{\prime}
$$

is holomorphic over $\Omega_{T}$ defined by

$$
\Omega_{T}=\left\{\left(z_{r}+i z_{i}, \theta_{r}+i \theta_{i}\right) \in \mathbb{C}^{2} \mid\left(z_{i}, \theta_{i}\right) \in \bigcap_{k \in \mathbb{Z}} \Omega_{T}\left(z_{r}+2 \pi k\right)\right\} .
$$




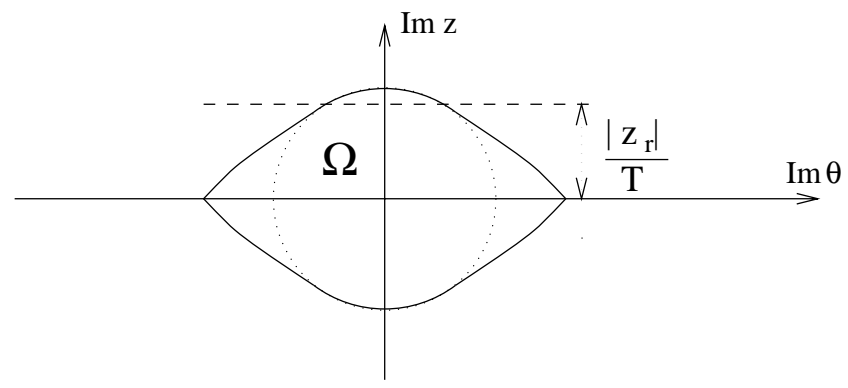

FiguRE 8 . The set $\Omega_{T}$.

So both components of $B S(g)(\theta, z)$, as defined by (4.2), are also holomorphic over $\Omega_{T}$.

Now for any real number $\epsilon$ in $(0,1), \Omega_{T}$ contains a neighborhood of the set of

$$
z=\pi, \theta \in i\left[0, \theta_{i}^{0}(0, \pi)-\epsilon\right]
$$

which, together with (4.8), proves Lemma 4.2.

\section{REFERENCES}

[1] B. Allibert, Contrôle analytique de l'équation des ondes sur des surfaces de révolution. C. R. Acad. Sci. Paris 322 (1996) 835-838.

[2] B. Allibert, Contrôle analytique de l'équation des ondes sur des surfaces de révolution. Thèse de doctorat de l'École Polytechnique (1997).

[3] A. Haraux, Séries lacunaires et contrôle semi-interne des vibrations d'une plaque rectangulaire. J. Math. Pures Appl. 68 (1989) 457-465.

[4] G. Lebeau, Control for hyperbolic equations. Journées "équations aux dérivées partielles" Saint Jean de Monts, 1992. École Polytechnique, Palaiseau (1992).

[5] G. Lebeau, Fonctions harmoniques et spectre singulier. Ann. Sci. École Norm. Sup. 13 (1980) 269-291.

[6] J.-L. Lions, Contrôlabilité exacte, perturbation et stabilisation des systèmes distribués. Rech. Math. Appl. 8-9 (Masson, Paris, 1988).

[7] F. Treves, Introduction to pseudodifferential and Fourier integral operators, New York and London Plenum Press Vol. 2, 25 $\mathrm{cm}$ (The university series in mathematics, 1980).

[8] A. Zygmund, Trigonometric Series, Cambridge Univ. Press (1968). 